\title{
Research Paper \\ Investigating the effectiveness of successful intelligence components training based on sternberg's triarchic theory on increasing fluid reasoning and verbal comprehension
}

\footnotetext{
Peyman Kamkar $^{1}$, Fariborz Dortaj ${ }^{2}$, Esmaeil Saedipour ${ }^{2}$, Ali Delavar ${ }^{3}$, Ahmad Borjali $^{4}$

1. Ph.D Student of Educational Psychology, Faculty of Psychology and Educational Sciences, Allameh Tabataba'i University, Tehran, Iran.

2. Professor, Department of Educational Psychology, Faculty of Psychology and Educational Sciences, Allameh Tabataba'i

University, Tehran, Iran.

3. Professor, Department of Assessment and Measurement, Faculty of Psychology and Educational Sciences, Allameh Tabataba'i University, Tehran, Iran.

4. Professor, Department of Clinical Psychology, Faculty of Psychology and Educational Sciences, Allameh Tabataba'i University, Tehran, Iran.
}

Citation: Kamkar P, Dortaj F, Saedipour E, Delavar A, Borjali A. Investigating the effectiveness of successful intelligence components training based on sternberg's triarchic theory on increasing fluid reasoning and verbal comprehension. J of Psychological Science. 2021; 20(104): 1251-1266.

URL: https://psychologicalscience.ir/article-1-1095-fa.html
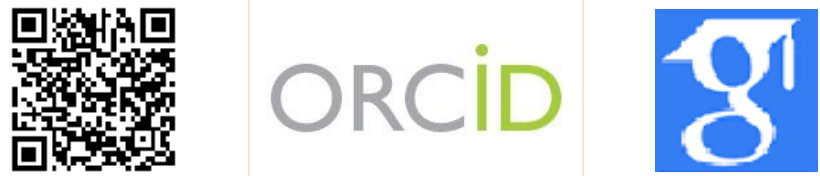

$10.52547 /$ JPS.20.104.1251

\section{A R T I C L E I N F O A B S T R A C T}

Keywords:

Teaching intelligence, Successful intelligence, fluid reasoning, verbal comprehension

Received: 24 Dec 2020 Accepted: 18 Jan 2021 Available: 23 Oct 2021
Background: Improving cognitive abilities is one of important goal for many educational systems. In this regard, successful intelligence theory has a variety of educational implications. Past researches has shown the effectiveness of training the components of successful intelligence in various variables, but in relation to the effectiveness of successful intelligence components training on increasing cognitive abilities, there is a research gap.

Aims: The aim of this study was to investigate the effectiveness of successful intelligence components training on increasing fluid reasoning and verbal comprehension of seventh grade students.

Methods: The present study is applied and semi-experimental in terms of outline research. The statistical population consisted of all seventh grade students in Holeilan city in the academic year of 2019-20. The study sample included 30 students who were selected by available sampling and, then, were assigned into two experiment and control groups (each group of 15) through random assignment method. After the pre-test, students of experimental group received 30 training sessions of the components of successful intelligence (researcher-made) and then, post-test data were collected. The Wechsler scale for children (WISC-5, 2014) was used to collect data. Descriptive (frequency, mean and standard deviation) and inferential (multivariate analysis of covariance) testes were used to analyze the data using SPSS software version 24.

Results: There was a significant difference between the experimental and control groups in terms of fluid reasoning and verbal comprehension $(\mathrm{p}<0 / 01)$.

Conclusion: Training in the components of successful intelligence can increase the scores of fluid reasoning and verbal comprehension. Thus, successful intelligence components training program can be used as an effective training method to increase fluid reasoning and verbal comprehension.

* Corresponding Author: Fariborz Dortaj, Professor, Department of Educational Psychology, Faculty of Psychology and Educational Sciences, Allameh Tabataba'i University, Tehran, Iran.

E-mail: dortajf@atu.ac.ir

Tel: (+98) 2148393257

2476-5740/ (C) 2021 The Authors. This is an open access article under the CC BY-NC-ND license

(https://creativecommons.org/licenses/by-nc/4.0/). 


\section{Extended Abstract}

\section{Introduction}

One of the important topics in psychology is intelligence and cognitive abilities. In this regard, Gottfredson and Saklofske (2009) believe that there is no more central subject in psychology than intelligence and intelligence testing. One of the most important conceptualizations of intelligence, is Cattell-Horn-Carroll (CHC) theory which is based on a psychometric approach. This theory is the basis for the formation of the most important tests of intelligence and considers basic cognitive abilities as the basis of intelligence differences (Bowden, 2013). Cattell-Horn-Carroll (CHC) theory, as the most comprehensive and most empirically supported psychometric theory (Mc Grew., 2005), on the structure of cognitive abilities, provides a map of all known abilities that can be used as a guide for research and practice (Flanagan and Harrison, 2012). Fluid reasoning (ability to reason in new situations without prior knowledge) and verbal comprehension (ability to understand and reasoning skills) are among the most important basic cognitive abilities which are the basis of many cognitive differences between individuals. Given the role that these abilities can play in the academic and non-academic success of individuals, one of the most fundamental questions that deserves scientific study is to what extent can we hope to develop these important structures through education?

There are contradictory views in this regard; on one side are Jensen (1969) and his followers, who believe that cognitive differences are inherent and that genetics forms the basis of individual differences (Rushton., 2000; Lynn., 2008; Johnson \& Bouchard., 2011; Harrison et al., 2014) and on the other side, there are researchers such as Sternberg who see the role of education more strongly and believe in the flexibility of intelligence and cognitive abilities (Nickerson, 2020; Westby., 2020; Ritchie \& Tucker., 2018; Botvinick \& Braver, 2015; Buschkuehl \& Jaeggi., 2010; Nisbett et al., 2012; Halpern, 2011; Borella et al., 2010 Dickens and Flynn, 2001; Sternberg, Grigorenko, \& Jarvin, 2001; Jaeggi et al.,
2008; Heinzel et al., 2014; Coloma et al., 2020; Karageorgos et al., 2020 and Carretti et al., 2014). Sternberg's Triarchic Theory (2020) is one of the most important theories in this field that has various implications for training, strengthening and enhancing cognitive abilities. This theory consists of three parts; in the first part of the theory, the mental mechanisms responsible for planning, executing, and evaluating intelligent behaviors are examined. The second part examines the relationship between intelligence and experience. In this part of the theory, two important processes are examined: (1) How to cope with new tasks or conditions, (2) How to automate information processing. The third part examines the relationship between intelligence and the environment. This part of the theory explains the three processes of adaptation, change and environment selecting. According to Sternberg, these abilities are flexible and can be improved through training and enrichment programs (Sternberg, 2020). Therefore, the present study seeks to answer the question of whether fluid reasoning and verbal comprehension which according to CHC theory (as the theory with the most empirical support) are considered to be the basis of many cognitive differences in individuals, can be developed by teaching and practicing the components of intelligence based on Sternberg's Triarchic Theory?

\section{Method}

This research was a semi-experimental study with pre-test, post-test design and control group. The study population included of all seventh grade students in Holeilan city in the academic year of 2019-20. The study sample included 30 students who were selected by available sampling and, then, were assigned into two experiment and control groups (each group of 15) through random assignment method. The experimental group received 15 training sessions of components of successful intelligence (two sessions weekly; each lasts for 2 hours), while the control group did not. The instrument of this research were The Wechsler scale for children (WISC-5, 2014). This program included concepts such as metacomponents, performance components, knowledge acquisition components, automatization of 


\section{Monthly Journal of Psychological Science}

information processing, analytical, creative and practical thinking skills.

For conducting research was received a letter from the management of exceptional education in Illam, and coordinated with the schools' management. In the first stage of the research, the objectives of the research were explained to the participants and they completed a written consent to participate in the research. The Wechsler scale for children were used as a pre-test to assessment cognitive abilities (fluid reasoning and verbal comprehension). In the next stage, the successful intelligence program (researcher-made) was implemented for the experimental group (The data related to the training program validation are presented in Table 1). After the intervention program, both experimental and control groups were retested using WISC-5. Analysis of Variance was used for analyzing the data.

\section{Results}

Descriptive indicators of fluid reasoning and verbal comprehension for the experimental and control groups in the pre-test and post-test are showed in Table 1. Findings implied that the mean score of fluid reasoning and verbal comprehension for both experimental groups increased at the posttest stage.

Multivariate analysis of covariance (MANCOVA) was used to evaluate the effectiveness of successful intelligence components training on increasing fluid reasoning and verbal comprehension. All assumptions of this test including normality of score distribution, homogeneity of group variances, linearity of synchronous and dependent variable, absence of outliers and homogeneity of variancecovariance matrix were examined and the results
Vol. 20, No. 104, Autumn(November) 2021

showed that all assumptions are valid. The results MANCOVA for fluid reasoning and verbal comprehension scores of the experimental and control groups are presented in Tables 2 and 3.

Based on the results of Table 3 , the values of Pillai's Trace (0.76), Wilkes' lambda (0.23), Hoteling Trace (3.24) and the Roy's largest root (0.26) are significant $(\mathrm{P}<0.001)$. The significance of these tests shows that there is a difference between the mean post-test scores of the experimental and control groups in at least one of the variables of fluid reasoning and verbal comprehension. These differences are presented in Table 3. As the results in Table 3 show, the results of multivariate analysis of covariance between the experimental and control groups in fluid reasoning scores $(\mathrm{P}<0.001, \quad \mathrm{~F}=58.28)$ and verbal comprehension $(\mathrm{P}<0.001, \mathrm{~F}<27.07)=)$ Shows a significant difference. In fact, teaching the components of successful intelligence has been effective in increasing fluid reasoning and verbal comprehension in the post-test stage. The magnitude of this effect is 0.69 for fluid reasoning and 0.59 for verbal comprehension.

After removing the effect of pretest, the adjusted mean of fluid reasoning scores of the experimental group participants in the posttest stage (M: 24.91; SD: 0.477 ) was higher than the control group (M: 19.35; SD: 0.477). Also, the verbal comprehension scores of the participants in the experimental group in the posttest stage (M: 23.39; SD: 0.516) were higher than the control group (M: 19.28; SD: 0.516). Therefore, it can be concluded that teaching the components of successful intelligence has an effect on increasing the scores of fluid reasoning and verbal comprehension of students.

Table 1. Descriptive indicators of fluid reasoning and verbal comprehension

\begin{tabular}{cccccc}
\hline \multirow{2}{*}{ Variables } & \multirow{2}{*}{ Test } & \multicolumn{2}{c}{ Experimental group } & \multicolumn{2}{c}{ Control group } \\
\cline { 3 - 6 } & & $\mathrm{M}$ & $\mathrm{SD}$ & $\mathrm{M}$ & $\mathrm{SD}$ \\
\hline \multirow{2}{*}{ fluid reasoning } & Pre-test & 18.00 & 1.96 & 17.40 & 2.69 \\
& Post-test & 25.20 & 2.78 & 19.06 & 2.96 \\
\multirow{3}{*}{ verbal comprehension } & Pre-test & 18.26 & 2.01 & 16.20 & 1.78 \\
& Post-test & 24.20 & 2.48 & 18.47 & 2.23 \\
\hline
\end{tabular}

Table 2. MANCOVA tests of fluid reasoning and verbal comprehension

\begin{tabular}{cccccc}
\hline Tests & Value & df & Eror df & F & Sig. \\
\hline Pillai's Trace & 0.766 & 2 & 25 & 40.81 & 0.001 \\
Wilkes' lambda & 0.234 & 2 & 25 & 40.81 & 0.001 \\
Hoteling Trace & 3.264 & 2 & 25 & 40.81 & 0.001 \\
Roy's largest root & 0.264 & 2 & 25 & 40.81 & 0.001 \\
\hline
\end{tabular}


Table 3. Analysis of covariance the effect of educational intervention on dependent variables

\begin{tabular}{ccccccc}
\hline Source of changes & Sum of squares & df & Mean square & F & Sig. & Effect size \\
\hline fluid reasoning & 198.17 & 1 & 198.17 & 58.28 & 0.001 & 0.69 \\
verbal comprehension & 93.71 & 1 & 93.71 & 27.08 & 0.001 & 0.51 \\
\hline
\end{tabular}

\section{Conclusion}

The present study was designed and conducted to investigate the effectiveness of successful intelligence components training on increasing fluid reasoning and verbal comprehension of seventh grade students. Findings showed that training the components of successful intelligence can increase the score of students' fluid reasoning (Consistent with the research of Nisbett et al., 2012; Borella et al., 2010; Jaeggi et al., 2008; Dickens and Flynn, 2001) and verbal comprehension (Consistent with the research of Coloma et al., 2020; Reynolds and Turek, 2012; Karageorgos et al., 2020; and Carretti et al., 2014).

Regarding the effectiveness of successful intelligence components training on increasing fluid reasoning, a large effect size was obtained, which indicates the high effectiveness of the training provided on increasing this ability. Fluid reasoning expresses the ability to reason in new situations without using prior knowledge, abstract reasoning, inferential reasoning, classification of stimuli into conceptual and conceptualization categories, and the ability to apply patterns and structures to visual-spatial information (Wechsler, 2014). Based on the evidence for Flynn effect, fluid intelligence is the most flexible dimension of intelligence. This dimension is also the highest predictor of academic and professional success. Therefore, it has become the first goal of interventions (Au et al., 2015). In this regard, Sternberg Kaufman and Grigorenko (2008) have introduced mechanisms through which individuals can solve a variety of problems (including problems of fluid reasoning). These mechanisms are: accurate definition of the problem, resource allocation, organizing the information, strategy development to solve the problem, strategy monitoring and evaluation of solutions, encoding, inference, and mapping.

Regarding the effectiveness of training the components of successful intelligence on increasing verbal comprehension, a high effect size was obtained, which indicates the high effectiveness of the training provided on increasing the ability of verbal comprehension. Verbal comprehension as the ability to understand and reasoning skills (Kan et al. 2011) is considered as a common reason for the observed differences between vocabulary scores, verbal skills and general verbal knowledge (McArdell et al. 2002). Research in this field shows that this ability can be developed and education can provide the ground for its growth (Koloma et al., 2020; Karageorgos et al., 2020). In this regard, Sternberg, Kaufman, and Grigorenko (2008) point to three important processes, including selective coding, selective synthesis, and selective comparison, through which individuals can develop new knowledge (including vocabulary and comprehension). Therefore, these processes have been considered in the training package used in the present study, and several activities have been considered to develop them; Therefore, it seems that the most important reason for better performance of the experimental group than the control group in posttest of verbal comprehension is doing these exercises.

The main finding of this study is that basic cognitive abilities are flexible, and can be increased in different ways. For example, Duke emphasizes the role of attitudes toward the changeability or immutability of cognitive abilities and he believes that people's attitude toward intelligence and cognitive abilities is one of the main factors in the growth and development of cognitive abilities and intelligence (Westby, 2020). Botvinik and Brewer (2015) highlighted the role of motivation in cognitive control using the evidence provided by neuroscience studies. Some have emphasized the importance of working memory in increasing intelligence and cognitive abilities and have provided evidence that they have been able to increase fluid and crystalline intelligence by practicing and strengthening working memory (Jacky et al., 2015). Ritchie and Tucker (2018) after reviewing 600,000 students in the form of a metaanalysis claimed that the results show that there is a 
direct positive relationship between academic years and intelligence. In addition, they claim that the results show that each academic year can increase the IQ score by between 1 and 5 points.

The training package used in this study is based on the conceptualization of intelligence by Sternberg et al. (2008). One of the important features of this conceptualization is its comprehensiveness. In this theory, different dimensions of intelligence including mental processes that are responsible for planning, executing and evaluating intelligent behaviors, the relationship between intelligence and the inner world and individual experiences, as well as the relationship between intelligence and the outside world are considered. Based on this and by modeling this theory, in this study, the comprehensiveness of the theory was maintained and for its different parts, various practices were included in the training package. It seems that the main reason for the success of this training package in increasing the score of fluid reasoning and verbal comprehension is its comprehensiveness.

In interpreting the research findings, it is necessary to consider its limitations. Since this study was conducted among seventh grade male students, caution should be exercised in generalizing its findings to other groups. The use of available sampling and the lack of a follow-up stage are other limitations of this study. It is suggested that the present study be repeated in other groups by removing the mentioned limitations. In addition, the effectiveness of the developed training package on other cognitive and emotional variables such as executive functions, academic self-efficacy and academic achievement should be examined. Another research suggestion is to compare the effectiveness of successful intelligence training package with other educational and cognitive interventions on cognitive and emotional variables. Based on the research findings, it is suggested that teachers, psychologists and counselors use this training package to increase students' fluid reasoning and verbal comprehension.

\section{Ethical Considerations}

Compliance with ethical guidelines: The authors extracted this article from the $\mathrm{PhD}$ dissertation of the first author, which approved under No 514 date, 07.01.2019 in the Faculty of Psychology and Educational Sciences, Allameh Tabatabai University, Tehran. The General Department of Education of Ilam Province have signed the permission to carry the research under No 36962 date, 08.08.2019. Ethical considerations like gaining the informed consent of the participants and the confidentiality of responses were considered in this research.

Funding: This study was conducted as a $\mathrm{PhD}$ thesis with no financial support.

Authors' contribution: The first author was the senior author, the second and third authors was the supervisors and the fourth and fifth authors was the advisory professors of the dissertation. Conflict of interest: The authors declare no conflict of interest for this study.

Acknowledgments: We would like to appreciate the General Department of Education of Ilam Province and the Department of Education of Holeilan city for helping in collecting samples and providing a suitable place for conducting research, as well as all the students who participated in this research. 
اثربخشى آموزش مؤلفههاى هوش موفق مبتنى بر نظريه سهوجهى استرنبر كى بر افزايش استدلال سيال و دركى كلامى

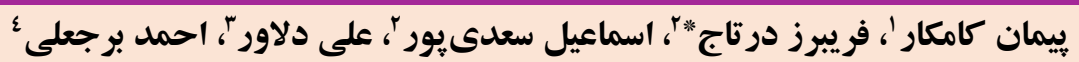

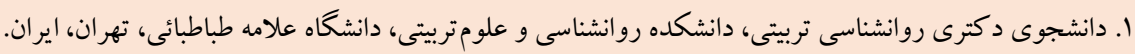

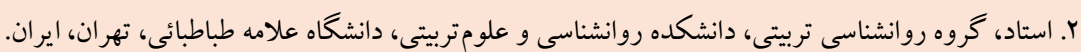

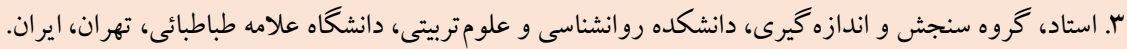

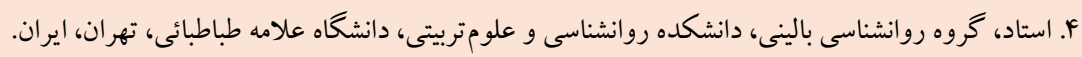

جكيده

زمينه: ارتقاء توانايىهاى شناختى ازجمله اهداف مهم براى بسيارى از نظامهاى آموزشى است. در اين زمينه نظريه هوش موفق آنق تلويحات

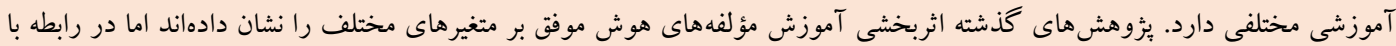
اثربخشى آموزش مؤلفهاى هوش موفق بر افز ايش توانايىهاى شناختى بايه خلاء يُروهشى وجود دارئ دارد.

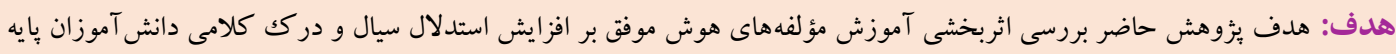
هفتم بود. روش: يُروهش حاضر كاربردى و از نظر طرح تحقيق شبه آزمايشى است. جامعه آمارى شامل كليه دانش آموزان پايه هفتم شهرستان هليلان

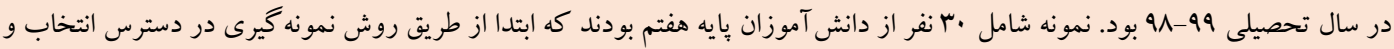

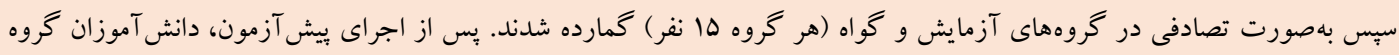

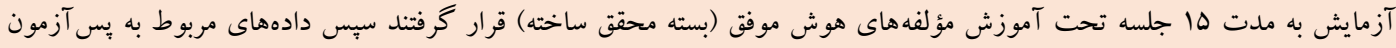

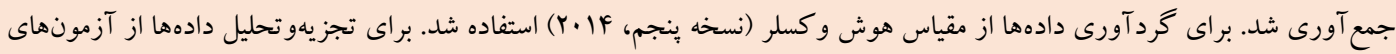

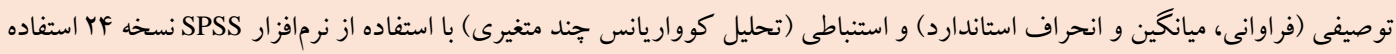

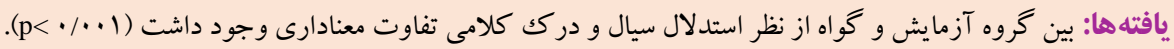

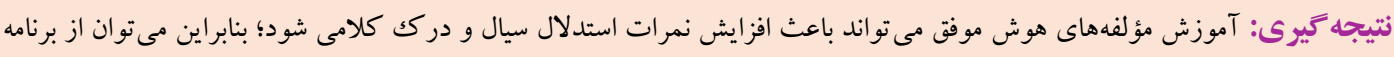

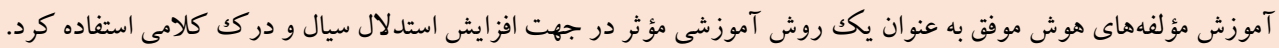

مشخصات مقاله

كليدوازهها:

آموزش هوش،

موش موفق،

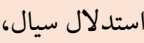

درك كلامى 


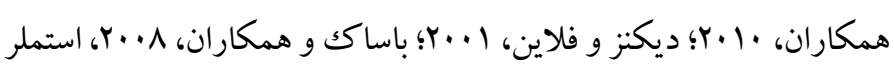

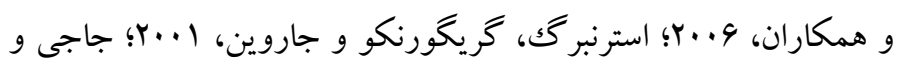

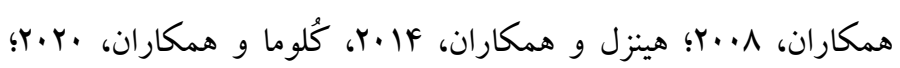

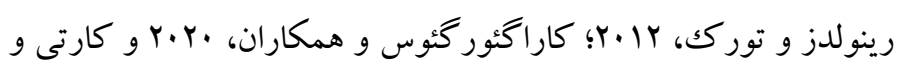

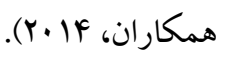

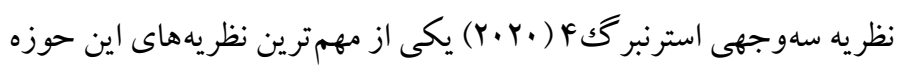

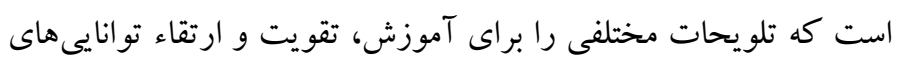
شناختى دارد. اين نظريه از سه بخش تشكيل مى شود؛ در بخش اول اول نظريه،

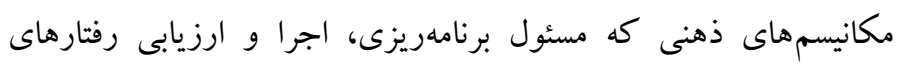

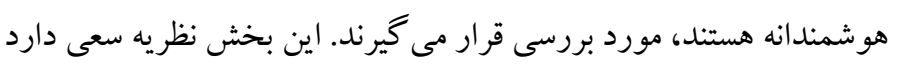
به اين سؤال بِاسخ دهد كه براى توليد رفتارهاى هوشمندانه، از كدام

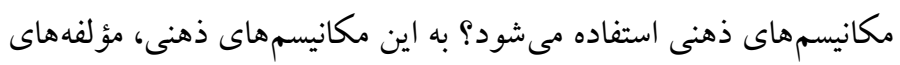

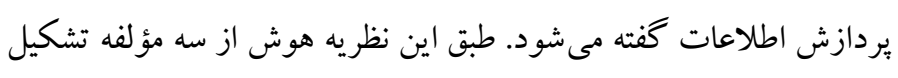

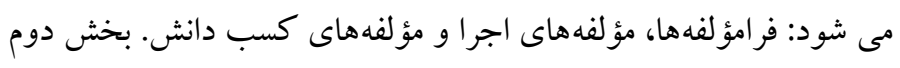

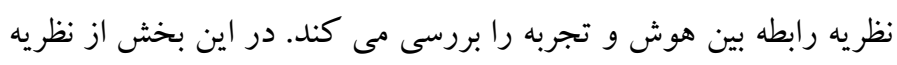

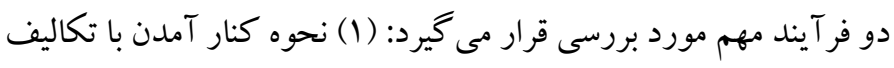

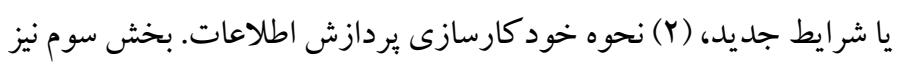
به بررسى رابطه بين هوش و محيط مى يردازد. اين بخش از نظريه به توضيح

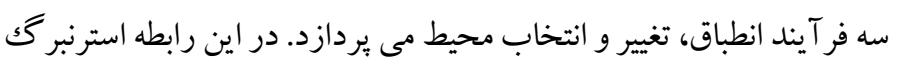

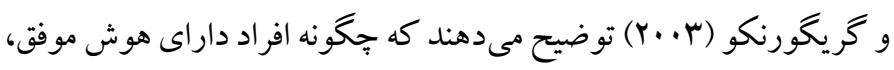
با استفاده از تعادلى كه ميان توانيى هاى تفكر تحليلى، خلاق و عملى بلى برقرار

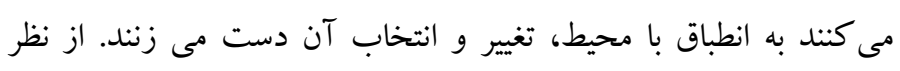

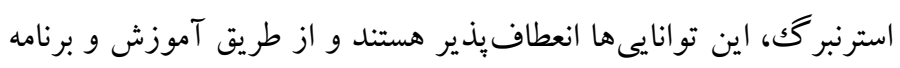

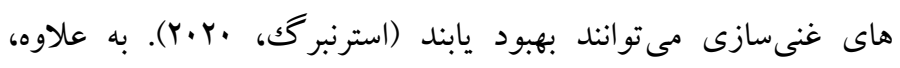

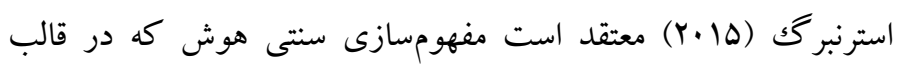

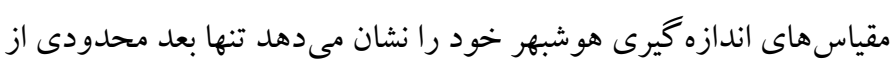
توانايى هايى كه براى موفقيت لازماند را اندازه گيرى مى كنند و قابليت شرح كامل سازه هوش را ندارند و نيز مدعى است نظريه او علاوه بر آنجه نظريه

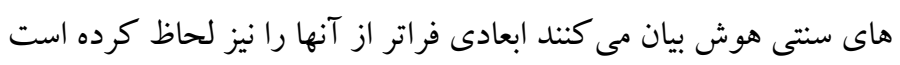

3 . verbal comprehension

${ }^{4}$. Sternberg's triarchic theory
مقالمه

يكى از موضوعات مهم علم روانشناسى هوش و تو انايى هاى شناختى است.

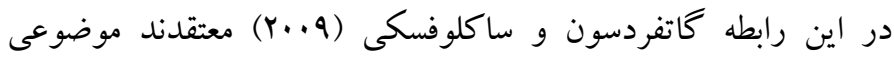
مر كزى تر از هوش و هوش آزمايى در روانشناسى وجود ندارد. درباره هوش و تو انايىهاى شناختى نظريهها و مفهومسازى هاى مختلفى ارائه شده است.

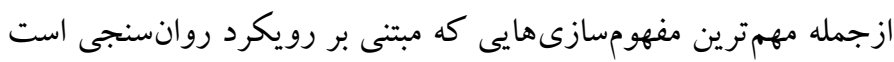

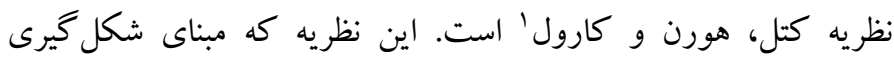

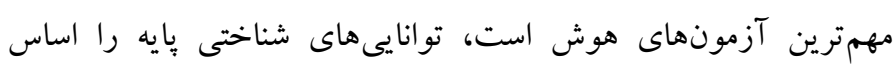

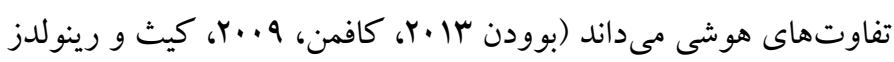

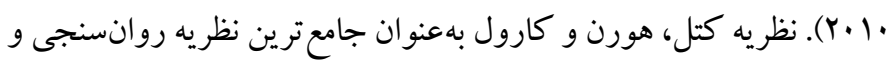

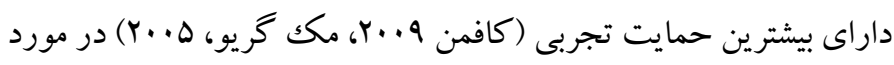
ساختار توانايى هاى شناختى يكك نقشه از همه توانايى هاى شناخته شده

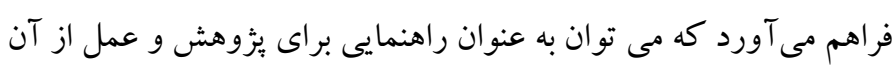

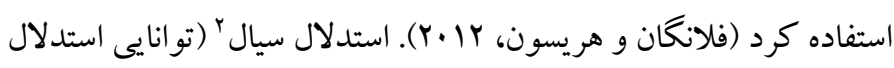

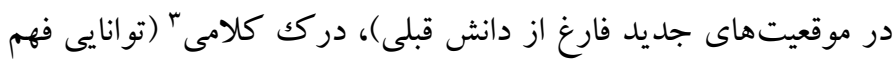

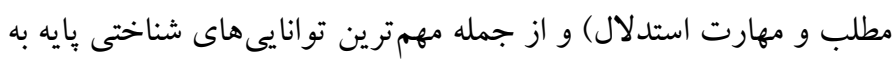

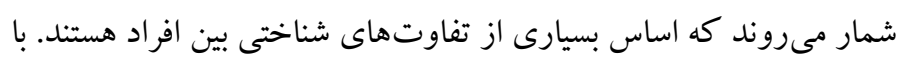

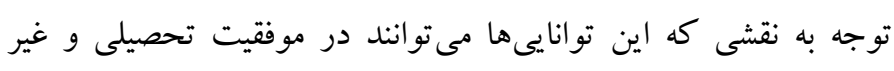

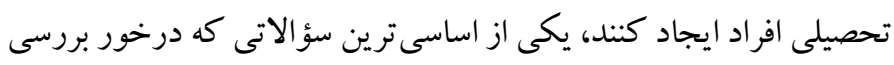

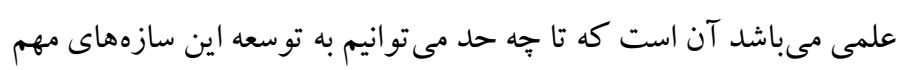

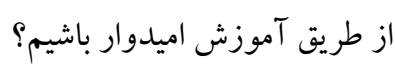
در اين رابطه ديد گاههاى متضادى وجود دارد؛ در يك طرف آن آرتور جنسن

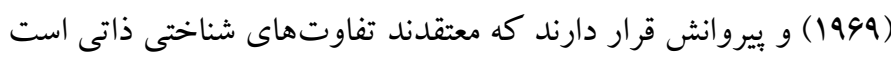

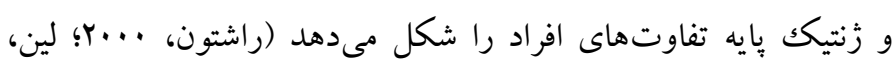

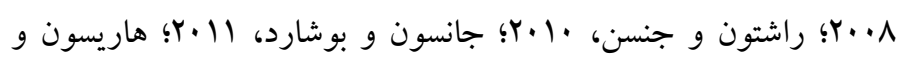

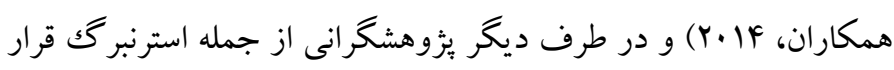

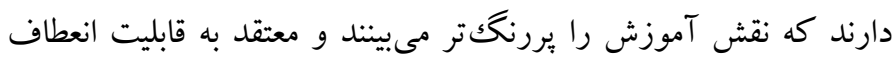

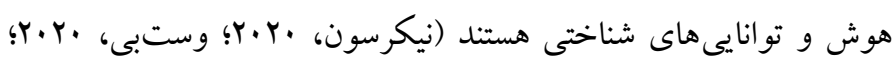

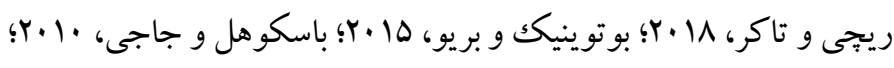

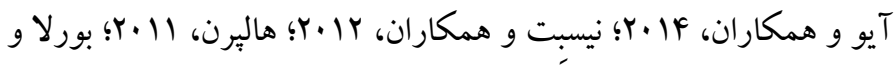

1. Cattell-Horn-Carroll (CHC) theory

${ }^{2}$. fluid reasoning 
هوش و كسلر متشكل از ه شاخص اصلى درك كلامى، ديدارى فضايى،

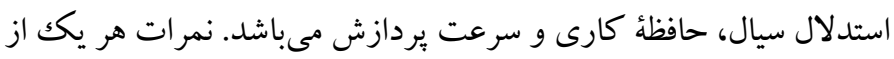

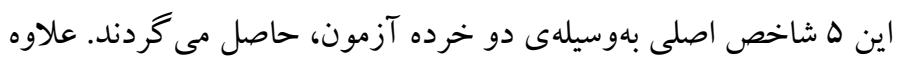

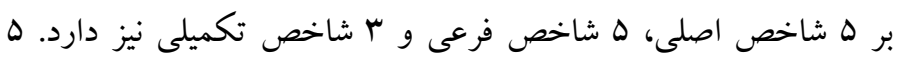
شاخص فرعى عبار تند از توانايى كلى، تبحر شناختى، غير كلاميى فئ، استدلال

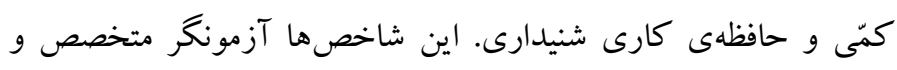

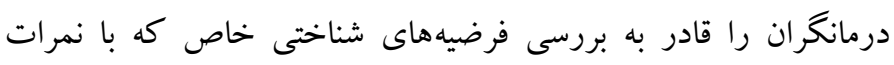

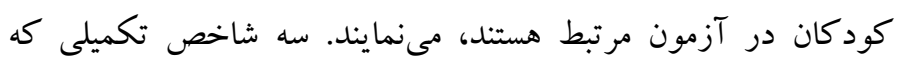

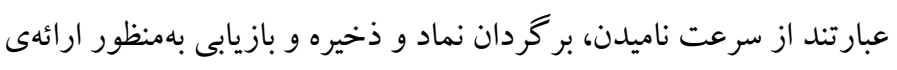

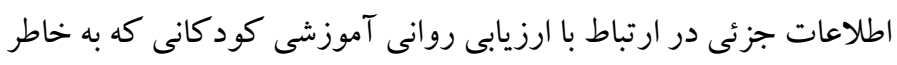

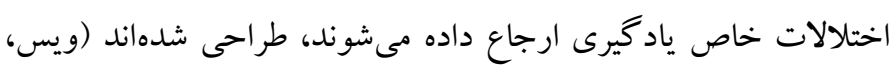

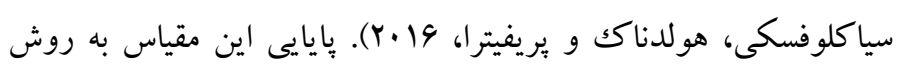

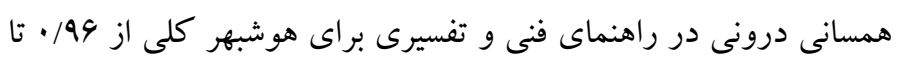

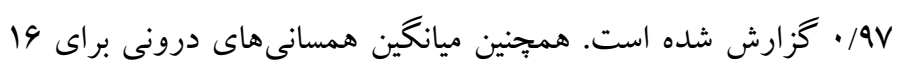

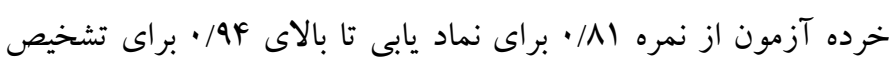

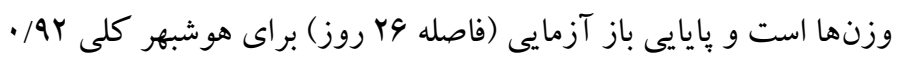

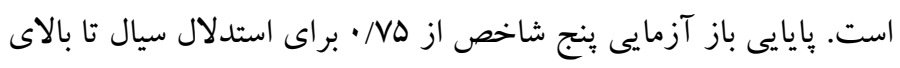

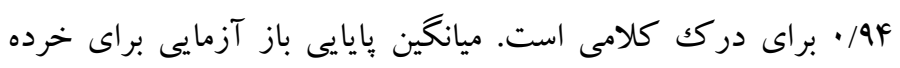

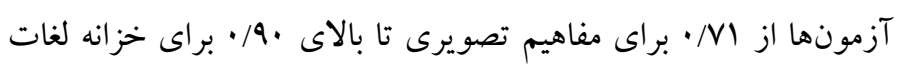

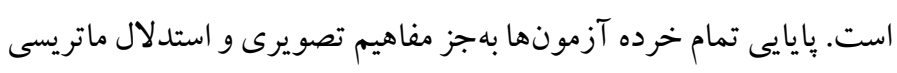

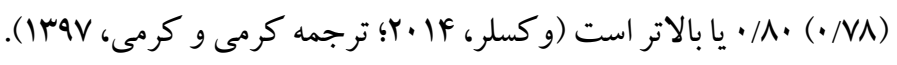
از سويى روايى ملاكى بر مبناى مجموعه ارزيابى كافمن براى كودكان

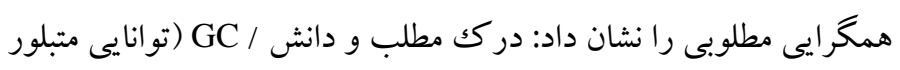

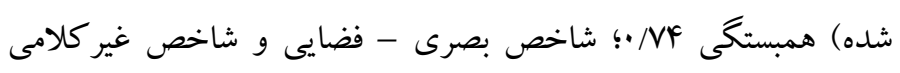

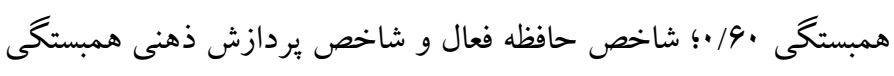

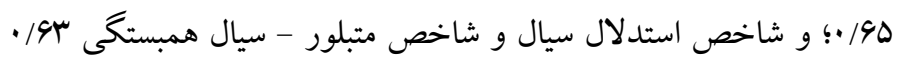

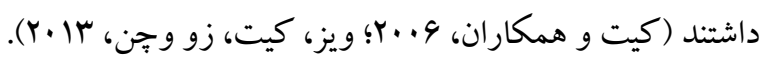

ج) برنامه مداخلهاى به منظور طراحى برنامه آموزشى مورد نظر، ابتدا نظريه مها، بُزوهشهاى

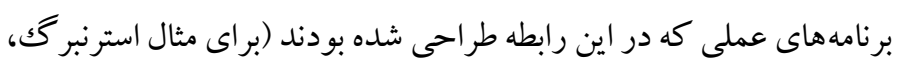

${ }^{2}$. Inclusion and exclusion criteria
و آنجه را كه افراد براى موفقيت در درس و زندگى واقعى لازم دارند شرح داده است.

بر اين اساس و باتوجه به اين حجم از اثربخشى ادعا شده مى توان بيشينى

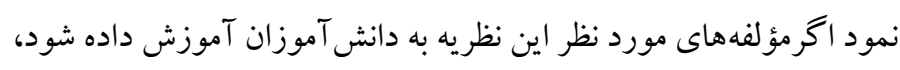

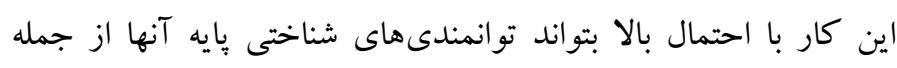

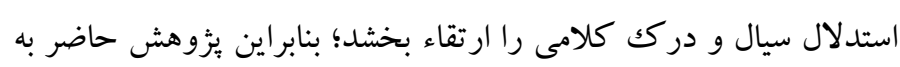

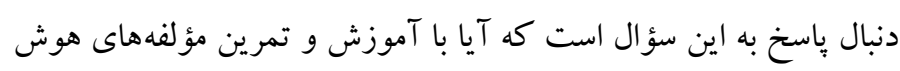

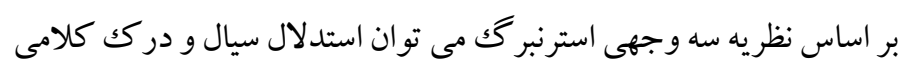
را كه بر اساس نظريه CHC (به عنوان نظريه داراى بيشترين حمايت تجربى)

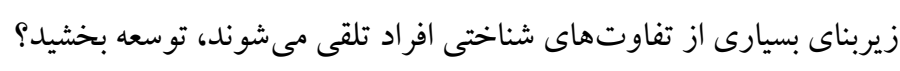

روش

الف) طرح ئوهش و شركت كنند كان: يُزوهش حاضر، يك مطالعه

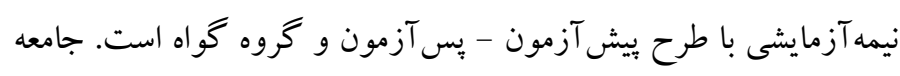

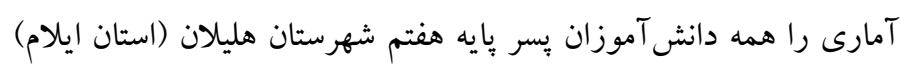

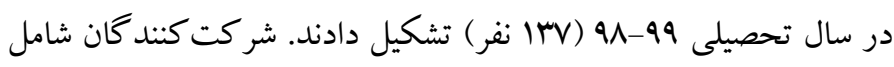
• ب نفر بودند كه به روش نمونه گيرى در دسترس' بر اساس ملاككهاى

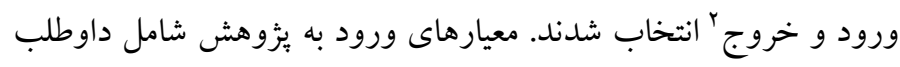
بودن دانش آموزان، نداشتن نقص جسمانى، دسترسى راحت به محل اجراى

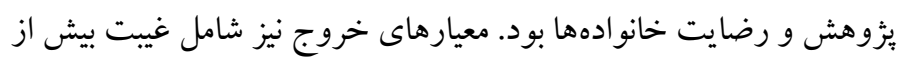

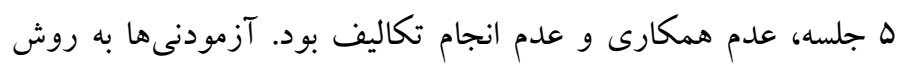

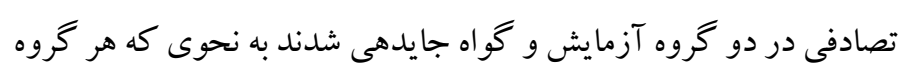
شامل ها نفر مى شد.

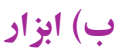

بهمنظور جمع آورى دادهها از مقياس هوش و كسلر كود كان نسخه بنجم إنما استفاده شد. اين مقياس كه آخرين نسخه از مقياس هاى و كسلر

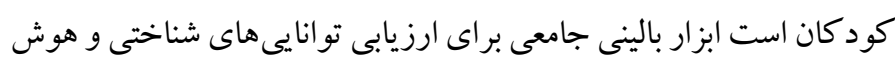

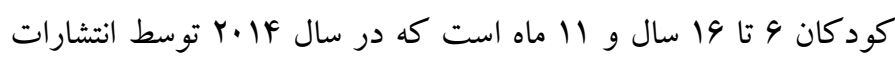
ييرسون ارائه شده. اين مقياس داراى اب خرده آزمون است كه در يزوهش

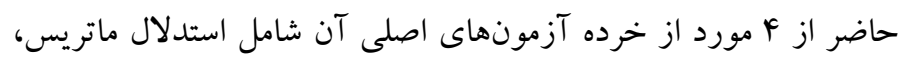

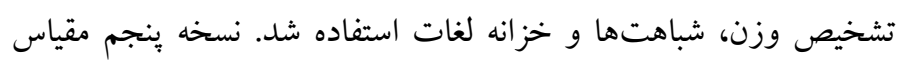

${ }^{1}$. convenience sampling 


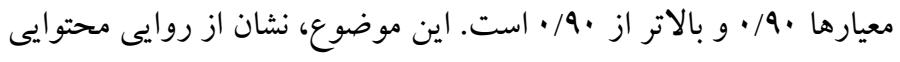

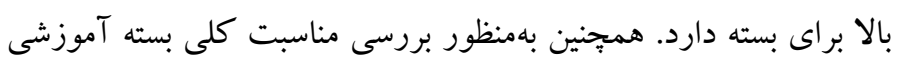
هوش موفق از ميانكين مجموع شاخص هاى روايى محتوايى استفاده شد.

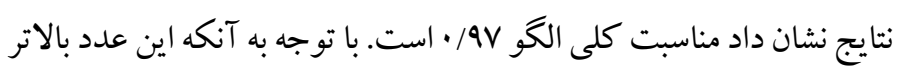

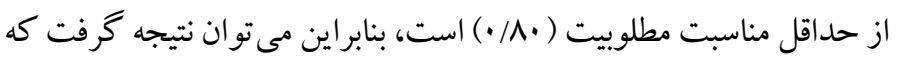

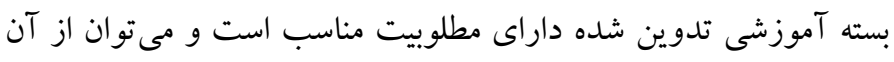
استفاده نمود. محتواى آموزشى جلسات در جدول بار ارائه شده است.

د) روش اجرا

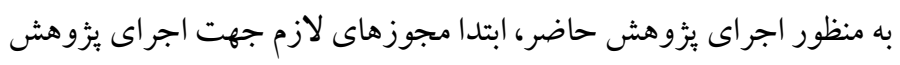

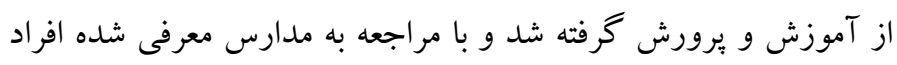

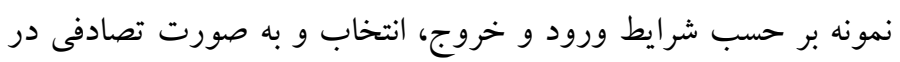

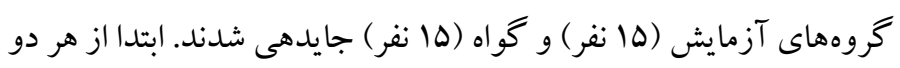

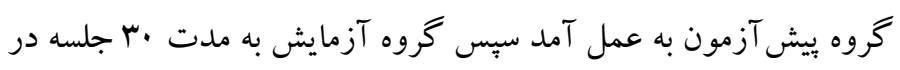

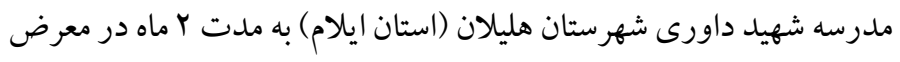

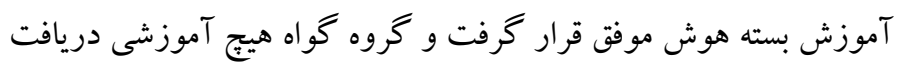

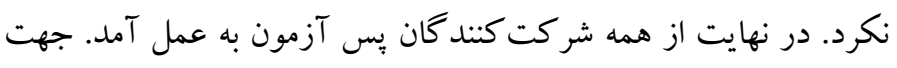

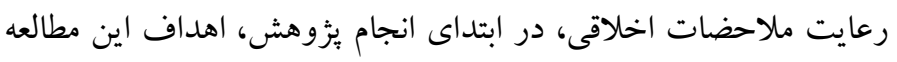

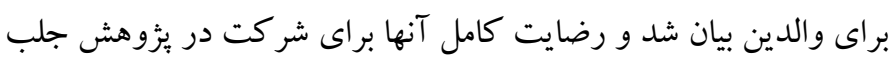

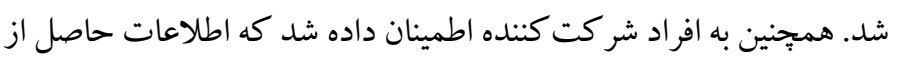
اين إزوهش به صورت گروهى مورد تحليل قرار گرفته و براى توليد

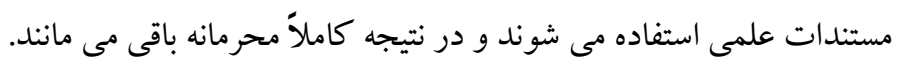

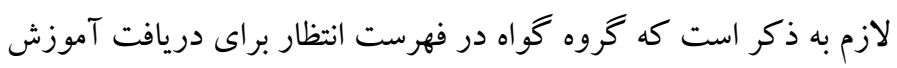

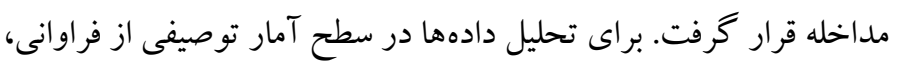

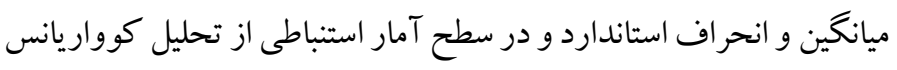

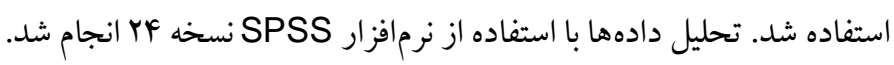

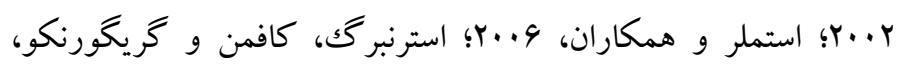

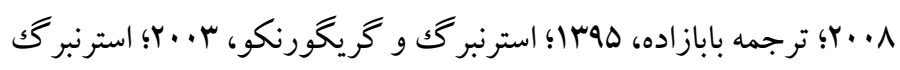

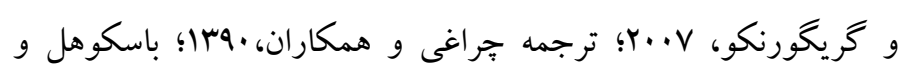

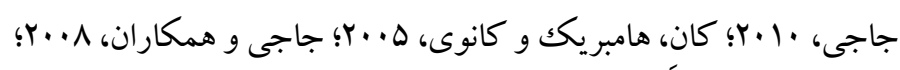

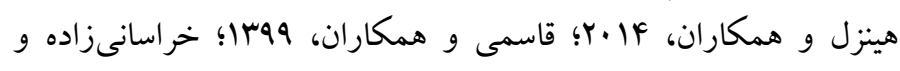

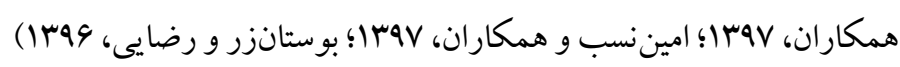
مطالعه و با جمع بندى آنها و نيز با لحاظ كردن شرايط فرهنكى كشور ايران

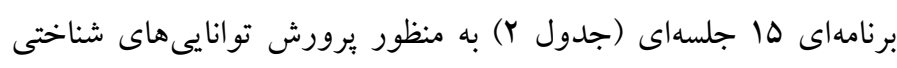

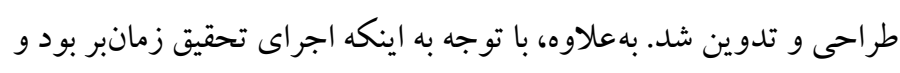

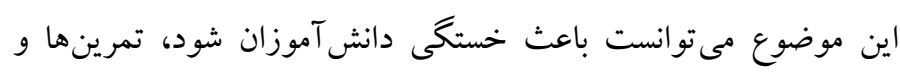
تكاليف بسته بيشتر در قالب بازى طراحى شدند. در طراحى و اجراى بـ إنى

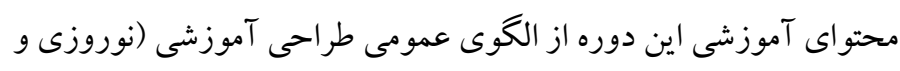
رضوى، ·وس|) استفاده شد. براى اعتباريابى بسته آموزشى از بانل متخصصين استفاده شد. به اين صورت

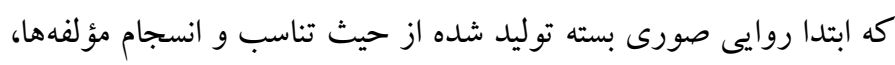

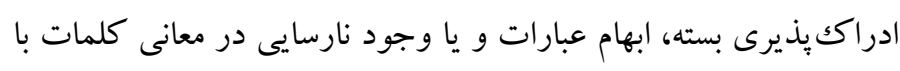
نظرات اساتيد راهنما و مشاور بررسى و اصلاح شد. سبس بيش بيشنويس اوليه

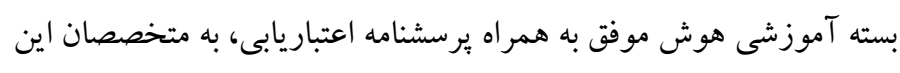

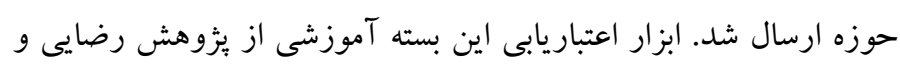

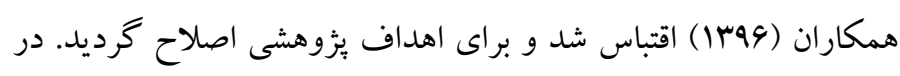

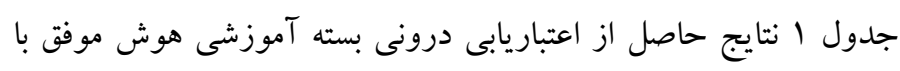

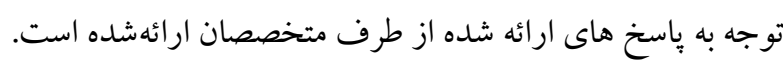

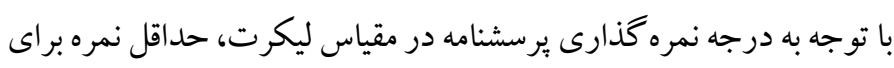

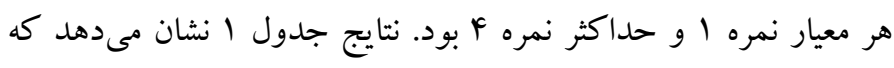

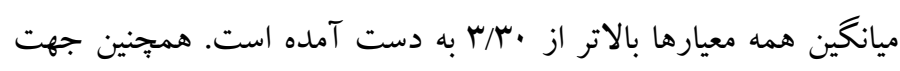
بررسى روايى محتوايى بسته بيشنهادى، از شاخص روايى محتوايى (CVI)

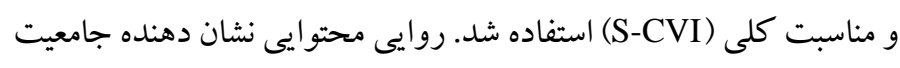

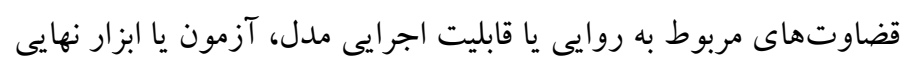

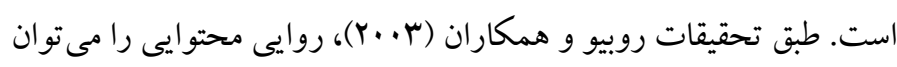

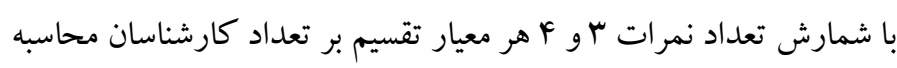

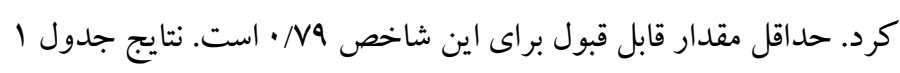

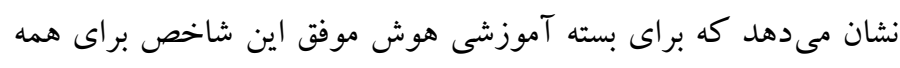


جدول ا. اعتباريابى درونى بسته آموزشى هوش موفق

\begin{tabular}{|c|c|c|c|c|c|c|c|}
\hline S-CVI & CVI & انحراف معيار & ميانگين & حداكثر & حداقل & معيار & رديف \\
\hline \multirow{7}{*}{.$/ 9 \mathrm{~V}$} & 1 & $\cdot / F \wedge$ & $r / v$. & $r$ & $r$ & اعتبار & 1 \\
\hline & 1 & $\cdot / \Delta r$ & $r / \Delta$. & F & $r$ & كاربرديذيرى & r \\
\hline & 1 & $\cdot / \Delta r$ & $r / 9$. & F & $r$ & انسجام & $r$ \\
\hline & 1 & $\cdot / 4 \wedge$ & $r / v$. & F & $r$ & جامعيت & r \\
\hline &.$/ 9$ & $\cdot / v$ & $r / 4$. & r & r & ادراككيذيرى & $\Delta$ \\
\hline & 1 & $.19 \mathrm{~V}$ & $r / r$. & F & r & نو آورى & 9 \\
\hline & 1 & . & $r / q$. & r & $r$ & مقبوليت & v \\
\hline
\end{tabular}

جدول r. شرح جلسات آموزش مؤلفه هاى هوش موفق

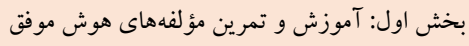

\begin{tabular}{|c|c|c|c|}
\hline تكاليف & محتوا & اهداف & جلسات \\
\hline 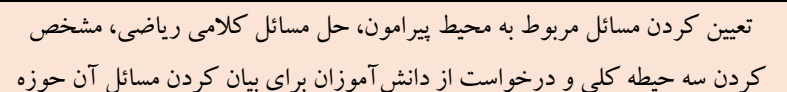 & داستان مرد جوب دبر، موارد لازم براى تعريف مسئه & آموزش فرا مؤلفه تعريف و & جلسه 1 \\
\hline 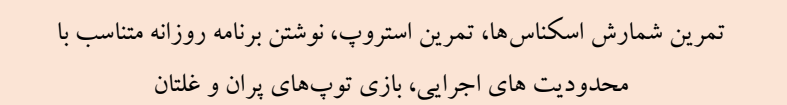 & 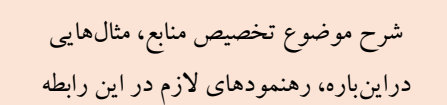 & آموزش فرا مؤلفه تخصيص & 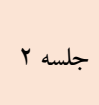 \\
\hline حل مسائل مربوط به رتبهندى افراد و اشياء، حركت بر روى صفحه شطرنجى، جور & شرح موضوع سازماندهى اطلاعات، آموزش & آموز مان فرا مؤلفه ارائه و & 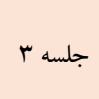 \\
\hline حل معماهاى كلامى، حل معماهاى بصرى، انجام بازى تا نخرام & آموزش تعيين مراحل حل مسئله، آموزش شيوه & استروز آتزى فرا مؤلفه تنظيم & 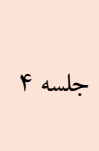 \\
\hline 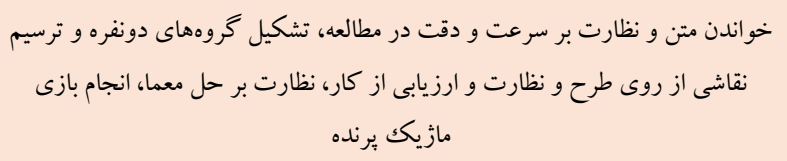 & 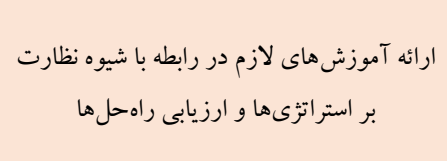 & آموزش فرا مؤلفه نظارت بر استرى و ارزيابى & جلسه هـ \\
\hline تقسيم مسائل گسترده به مسائل كو خجكت و و رمز كردانى آنها & آموزش نحوه رمز كردانى مسائل مختلف & آموزش مؤلفه اجرايى & 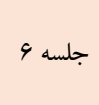 \\
\hline تكميل جدول روابط دو كانه & تركيب، ريز طبقه و....) بين اشياء، كلمات و و رختلف (تشابه، تضاد، & آموزش مؤلفه اجرايى استباط & 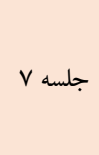 \\
\hline تكميل جدول روابط بين روابط دو كانه & آموزش استنباط روابط جند كانه بين رابطههاى دوبخشى & آموزش مؤلفه اجرايى نقشه & 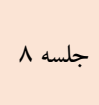 \\
\hline حل مسائل قياسى، حل مسائل تكميل سرىها، حل مسائل ماتريسى، حل مسائل & ارائه تمرينهاى مختلف در جهت تقويت & تمرين مؤلفههاى اجرايى & 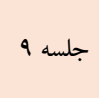 \\
\hline 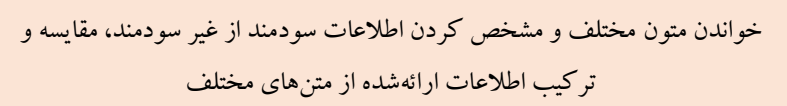 & آموزش نحوه رمز كردانى انتخابى، مقايسه & آموزش مؤلفهاى كسب & 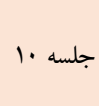 \\
\hline 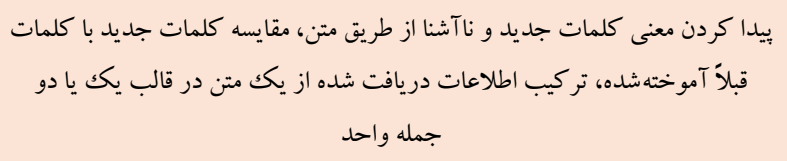 & تمر ين مهارتهاى سه كانه رمز كردانى انتخابى، & مؤ تمهين عملى به كارگيرى كسب دانش & 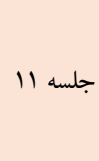 \\
\hline \multicolumn{4}{|c|}{ بخش دوم: خو دكارسازى يردازش اطلاعات } \\
\hline انجام تمرين سر كاف گذارى براى كلمات & توضيح و تبيين نحوه خود كارسازى، توضيح & $\begin{array}{l}\text { آموزش خود كارسازى } \\
\text { اطلاعات }\end{array}$ & جلسه r| \\
\hline
\end{tabular}




\begin{tabular}{|c|c|c|c|}
\hline \multicolumn{4}{|c|}{ بخش اول: آموزش و تمرين مؤلفههاى هوش موفق } \\
\hline تكاليف & محتوا & اهداف & جلسات \\
\hline 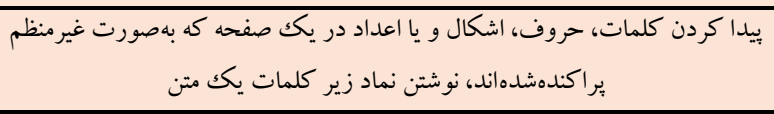 & خو ار كائه تمرين هاى مختلف مربوط به يردازش اطلاعات & $\begin{array}{l}\text { تمرين عملى خود كارسازى } \\
\text { اطلاعات }\end{array}$ & جلسه سו \\
\hline \multicolumn{4}{|c|}{ بخش سوم: آموزش مهارتهاى سه كانه تفكر (تحليلى، خلاق و عملى) } \\
\hline 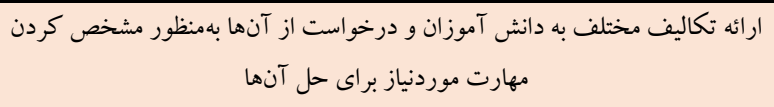 & 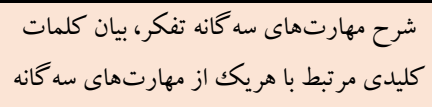 & توضيح و تبيين مهارتهاى سانه تفكر & If If \\
\hline 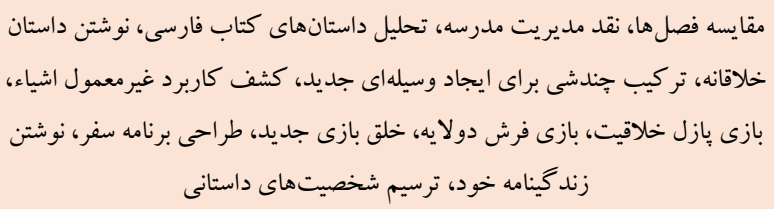 & 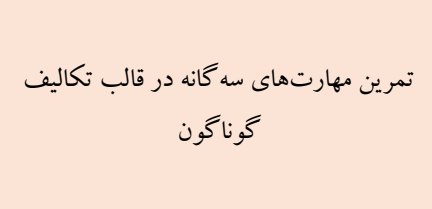 & تمرين مهارتهاى سه گانه & جلسه 10 \\
\hline
\end{tabular}

مقدار F متغير همكام محاسبه شد كه اين شاخص معنادار بود (ه •/P<)؛ : (P<) بنابر اين اين بيشفرض نيز رعايت شده است.

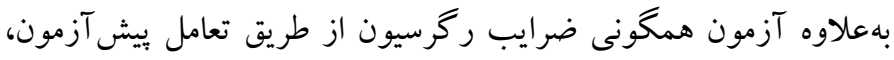

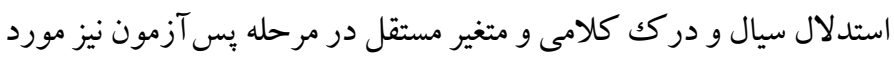

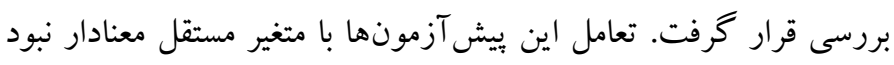

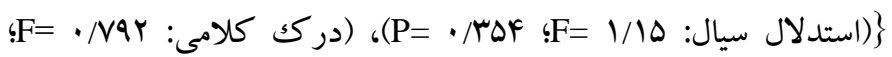

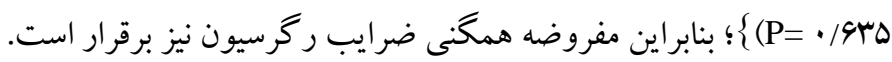
درنهايت براى بررسى بيشفرض همخنى ماتريس واريانس - كوواريانس

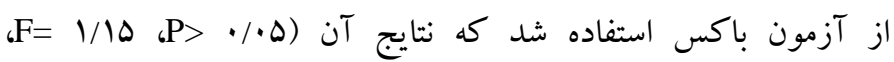
نشان داد اين بيش فرض رعايت شده است. (Box= r/VF.

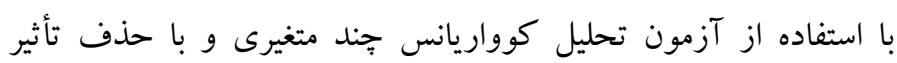

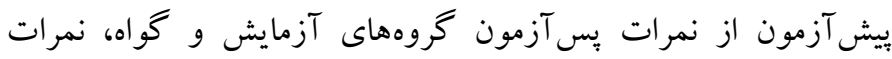

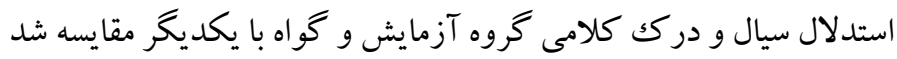

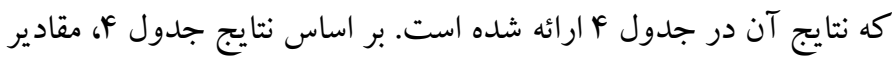

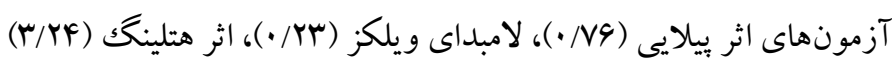

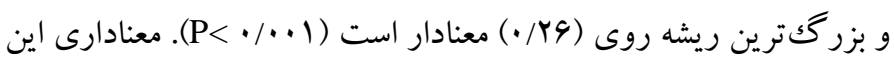
آزمونها نشان مىدهد كه حداقل در يكى از متغيرهاى استدلال سيال و

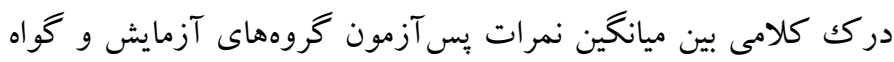
تفاوت وجود دارد. بررسى اين تفاوتها در جدول هـ هارائه شده است إن.

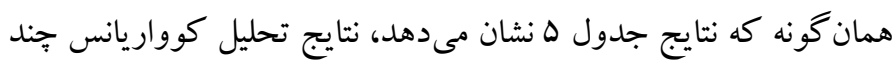

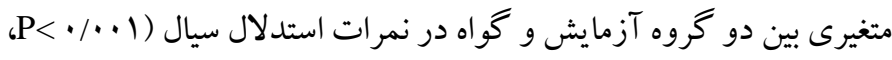
(F= DN/TA نشان مىدهد. درواقع آموزش مؤلفههاى هوش موفق بر افزايش توانمندى
يافته ها ميانگين و انحر اف استاندارد استدلال سيال و در كك كلامى شر كت كنند كان بر حسب مر حله و عضويت گروهى در جدول با ارائه شده است. همانطور

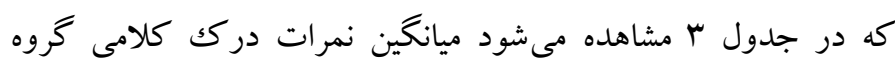

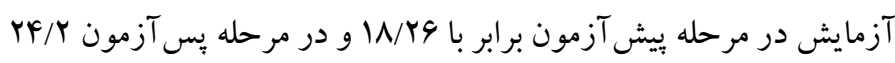

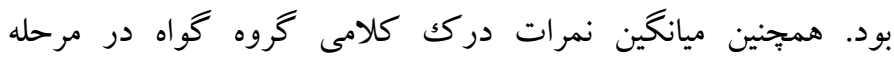

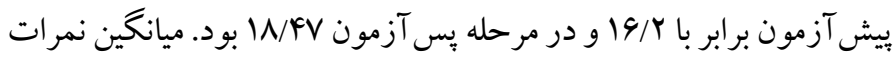

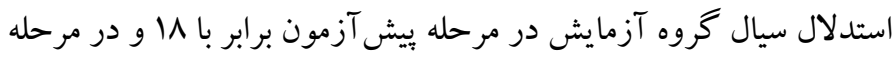

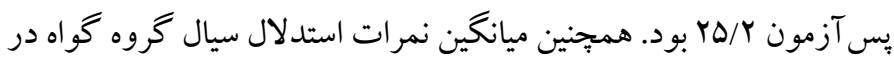

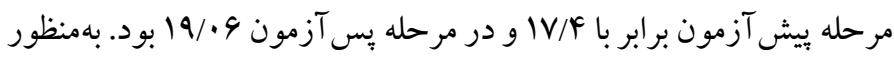

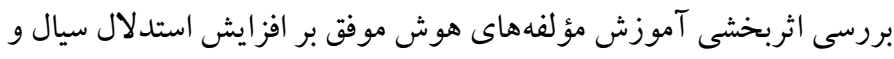

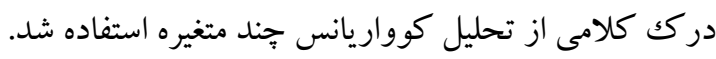

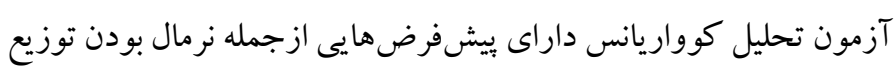

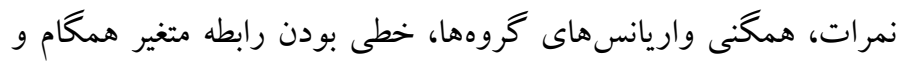

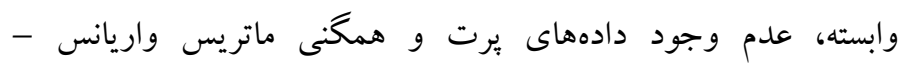

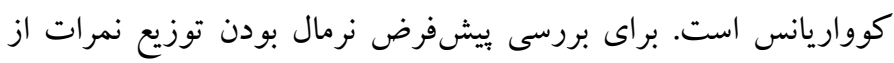

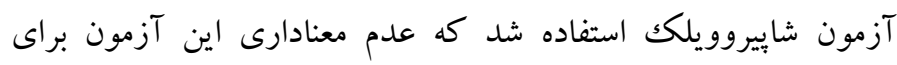

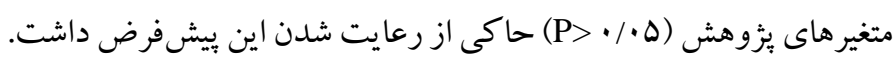

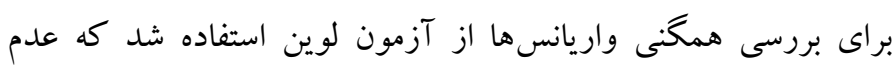

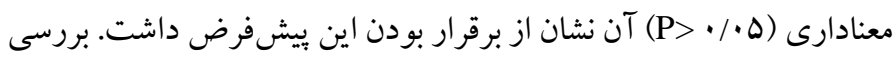

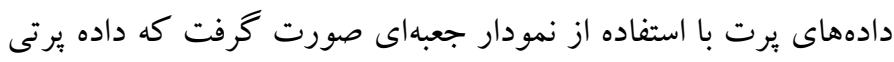

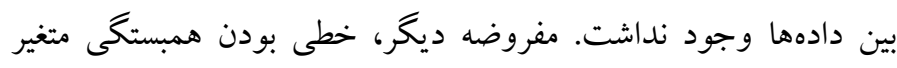
همگام و متغير مستقل است كه در اين يزوهش براى اثبات خطى بودن، 
و نيز نمرات دركك كلامى شركت كنندكان گروه آزمايش در مرحله

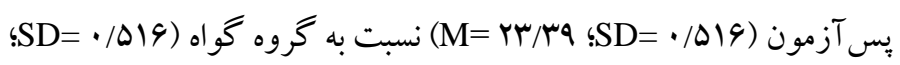

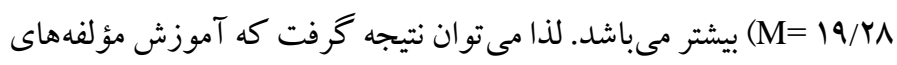
هوش موفق بر افزايش نمرات استدلال سيال و دركك كلامى دانش آموزان تأثير دارد.
شناختى استدلال سيال و دركك كلامى در مرحله بِ آزمون مؤثر بوده

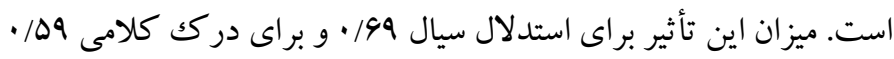

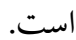
يس از حذف اثر ييش آزمون، ميانخين تعديل شده نمرات استدلال سيال

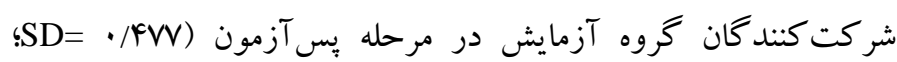

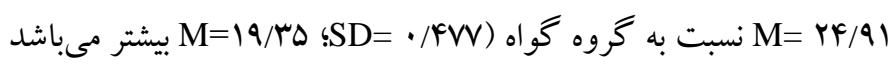

\begin{tabular}{|c|c|c|c|c|c|}
\hline \multicolumn{2}{|c|}{ يس آزمون } & \multicolumn{2}{|l|}{ ييش آزمون } & \multirow{2}{*}{ كرو } & \multirow{2}{*}{ متغيرها } \\
\hline انحراف استاندارد & ميانگين & انحراف استاندارد & ميانگين & & \\
\hline r/VA & $r \Delta / r$ & $1 / 99$ & 11 & آزمايش & \multirow{2}{*}{ ستدلال سيال } \\
\hline r/৭९ & $19 / .9$ & $r / 99$ & IV/F. & كواه & \\
\hline$r / F \Lambda$ & $r F / r$ & $r / \cdot 1$ & IN/YG & آزمايش & \multirow{2}{*}{ ركى كلامى } \\
\hline$r / r r$ & IN/FV & I/VA & $19 / 4$ & كواه & \\
\hline
\end{tabular}

\begin{tabular}{|c|c|c|c|c|c|}
\hline سطح معنادارى & خطbا df & فرضيه df & $\mathrm{F}$ & مقدار & نام آزمون \\
\hline.$/ \cdot 1$ & ro & r & $F \cdot|\lambda|$ &.$/ V 94$ & اثر ييلايى \\
\hline.$/ \cdot 1$ & ro & r & $F \cdot|\lambda|$ & . / TMF & لامبداى ويلكز \\
\hline.$/ \cdot 1$ & ro & r & $F \cdot|A|$ & r/YGA & اثر هتلينكگ \\
\hline.$/ \cdot 1$ & ro & r & $F \cdot / \lambda \mid$ & - /TSF & بزرگك ترين ريشه روى \\
\hline
\end{tabular}

\begin{tabular}{|c|c|c|c|c|c|c|}
\hline اندازه اثر & سطح معنادارى & $\mathrm{F}$ & ميانگين مجذورات & درجهآزادى & مجموع مجذورات & منبع تغييرات \\
\hline$\cdot 199$ & $\cdot / \cdot \cdot 1$ & $\Delta \Lambda / Y \Lambda$ & $191 / 1 V$ & 1 & $191 / 1 V$ & استدلال سيال \\
\hline$\cdot / \Delta 1$ &.$\cdots 1$ & $\mathrm{rV} / \cdot \mathrm{A}$ & $a r / v i$ & 1 & $a r / v i$ & درك كلامى \\
\hline
\end{tabular}

در رابطه با اثربخشى آموزش مؤلفهاى هوش موفق بر افزايش نمره توانايى

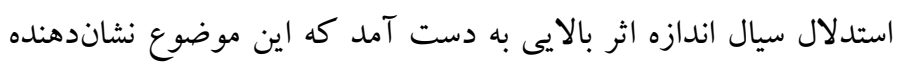
اثربخشى بالاى آموزش ارائه شده بر افزايش اين توانايى است. استدلال

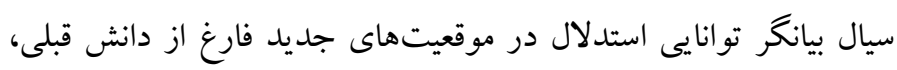

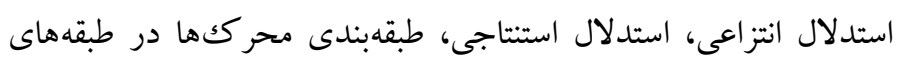

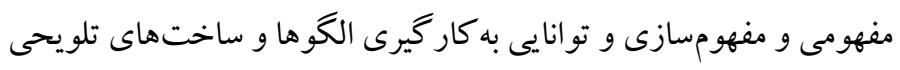

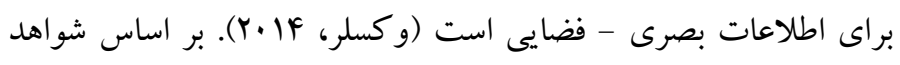
مربوط به اثر فلاين و نيز كارهاى تجربى صورت گرفته، هوش سيال

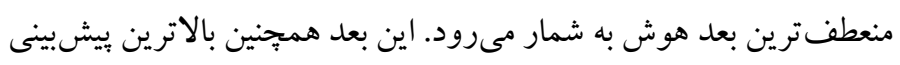
كننده موفقيتهاى تحصيلى و تخصصى است. از همين روى، به اولين

\section{بحث و نتيجه كيرى} يُزوهش حاضر بهمنظور بررسى اثربخشى آموزش مؤلفه هاى هوش موفق بر

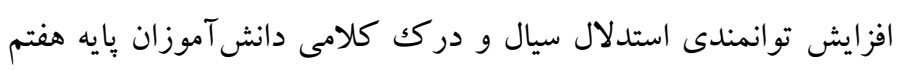

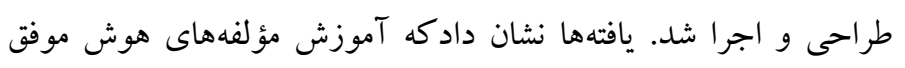

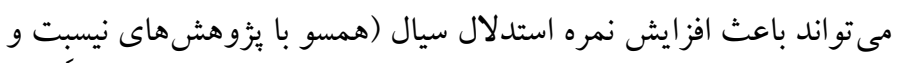

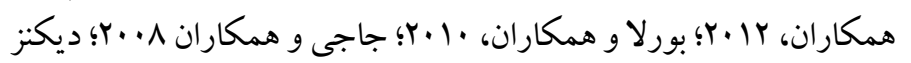

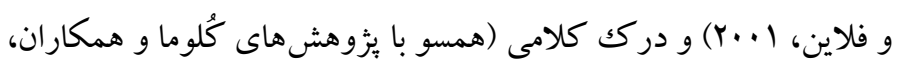

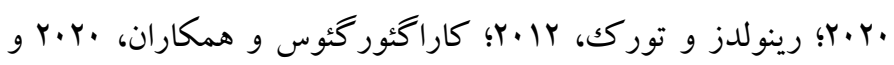

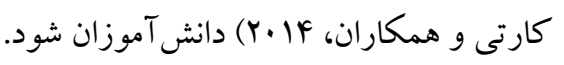


شناختى بررنگك كردهاند. عدهاى نيز بر اهميت حافظه كارى در افزايش هوش و توانايىهاى شناختى تأكيد كردهاند و شواهدى ارائه كردهاند كه بردي

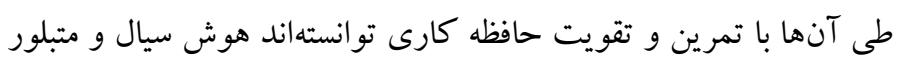

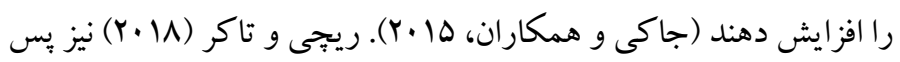

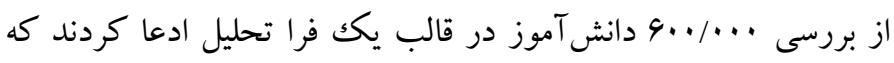
نتايج نشان مى دهد بين سالهاى تحصيلى و هوش يكك رابطه مثبت مستقيم وجود دارد. علاوه بر اين، آنها مدعى شدهاند كه نتايج نشان مىدهد هرسال تحصيلى مى تواند افزايشى بين ا تا ها درجه در نمره IQ ايجاد نماد نمايد.

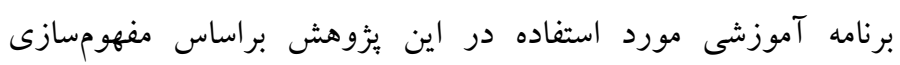

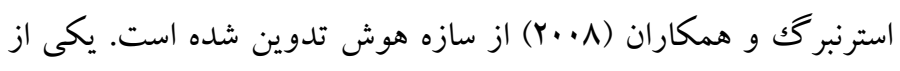

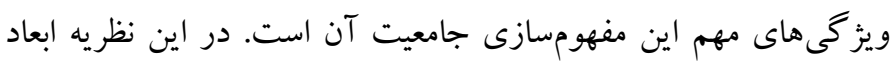

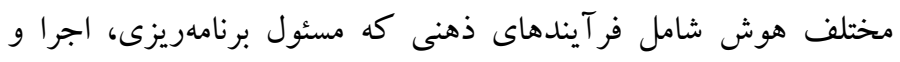
ارزيابى رفتارهاى هوشمندانه هستند، رابطه هوش و دنياى درونى و تجريبات

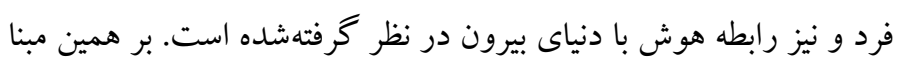

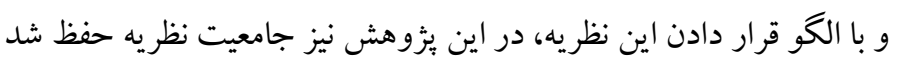

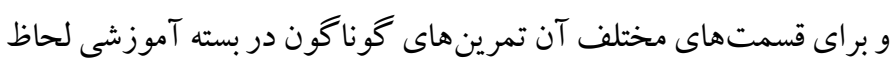
شد. به نظر مىرسد عمدهترين دليل موفقيت اين بسته آموزشى در افزايش

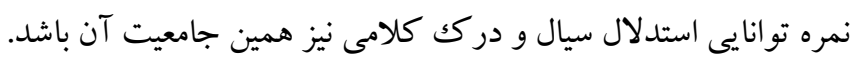
در تفسير يافتهاى بزوهش لازم است محدوديتهاى آن را نيز در نظر

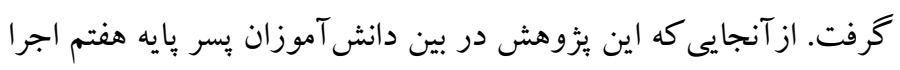

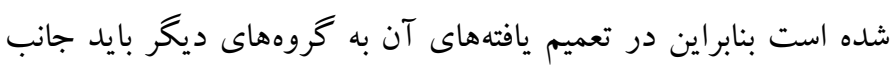

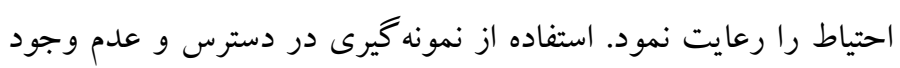

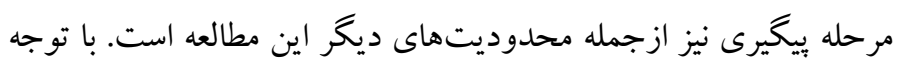

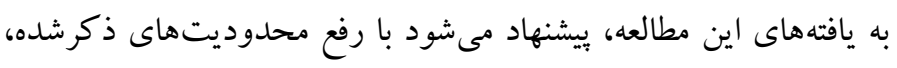

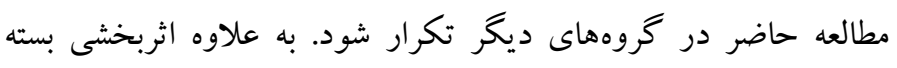
آموزشى تدوين شده بر متغيرهاى شناختى و عاطفى ديخر از قبيل

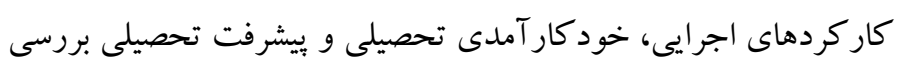

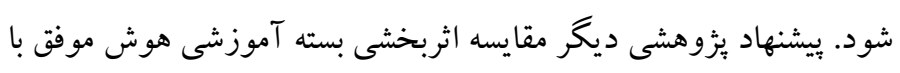

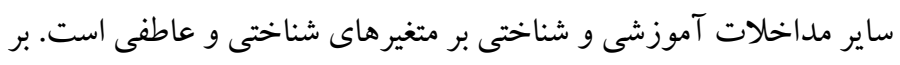

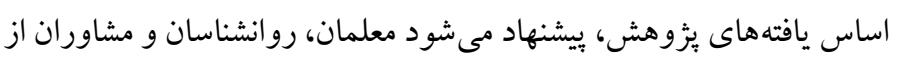
اين بسته آموزشى در جهت تقويت و افزايش توانايى استدلال سيال و درك كلامى دانش آموزان استفاده نمايند.
هدف مداخلات تبديل شده است (آيو و همكاران، F| • (Y). در اين رابطه

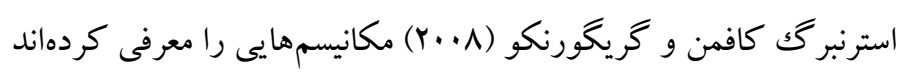
كه از طريق آنها افراد مىتواند انواع مسائل (ازجمله مسائل مربوط بهوريه

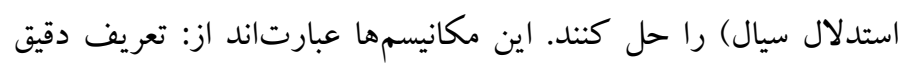
مسئله، تخصيص منابع، سازماندهى اطلاعات، تنظيم استراتزى براى حل

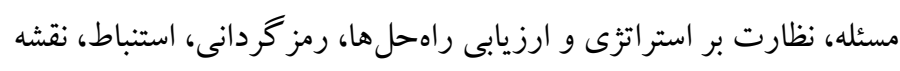
يابى است. در رابطه با اثربخشى آموزش مؤلفه هاى هوش موفق بر توانايى لريى

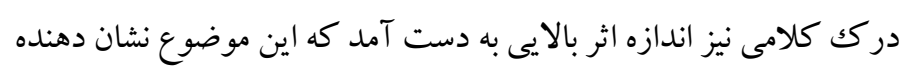
اثربخشى بالاى آموزش ارائهده بر افزايش توانايى درك كلى كلامى است.

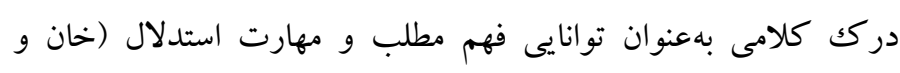

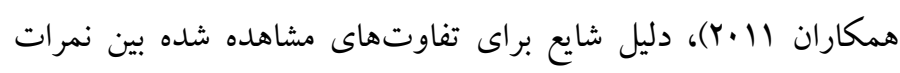
وازه گان، مهارت كلامى و دانش كلامى عمومى در نظر گرفته مى شود

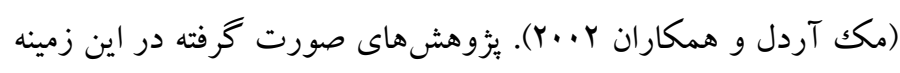
نشان مىدهند كه اين توانيى قابليت بسط و توسعه دارد و از طريق آموزش

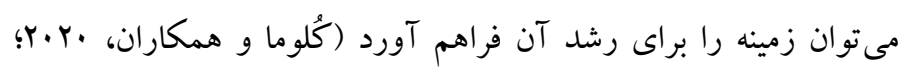

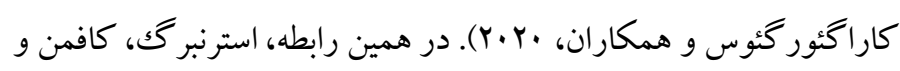

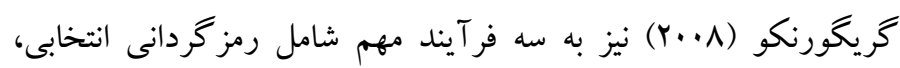
تركيب انتخابى و مقايسه انتخابى اشاره مى كنند كه از طريق آنها افراد

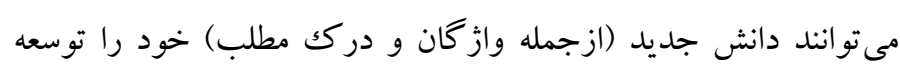

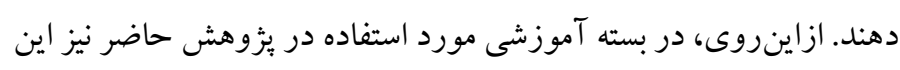

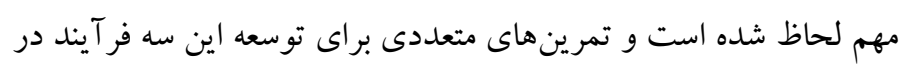

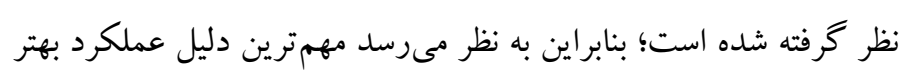

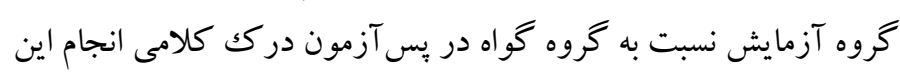
تمرين ها است. يافته اصلى اين يزوهش آن است كه توانمندىهاى شناختى بايه، قابليت

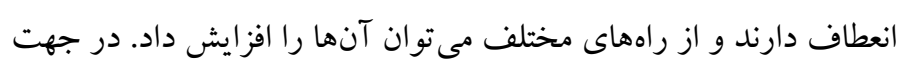

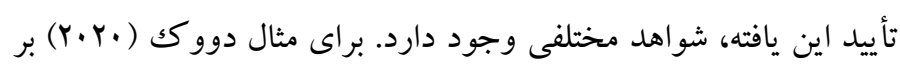

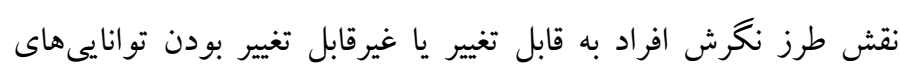

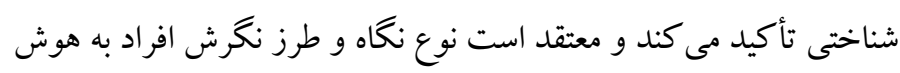
و تو انايى هاى شناختى يكى از عمدهت ترين عوامل رشد و تو سعه تو انايىهاى

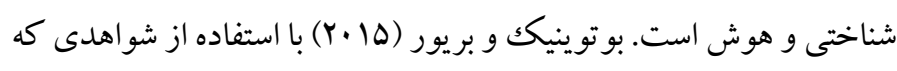

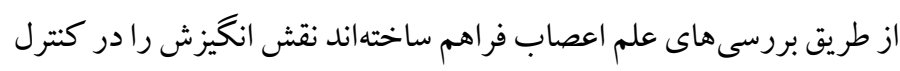


نقش هر يك از نويسند كان: نويسنده اول محقق اصلى اين يزوهش است. نويسنده

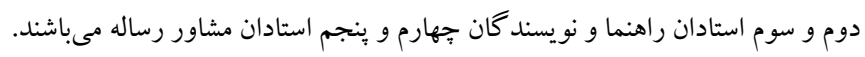

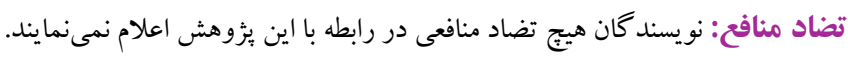

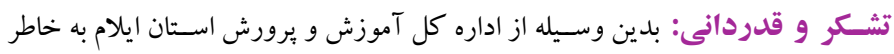

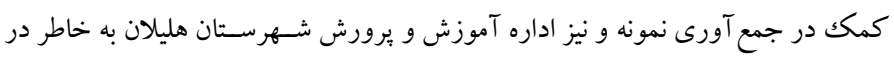

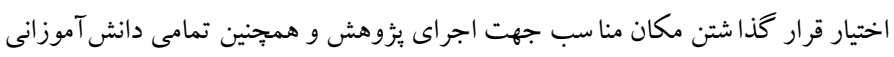
كه در اين يُووهش شركت كردند تشكر و قدردرانى مى شئود.

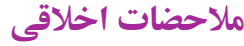

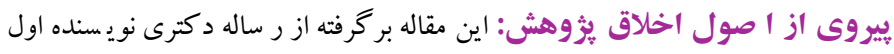

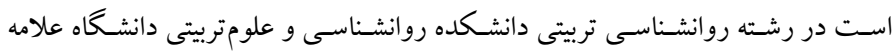

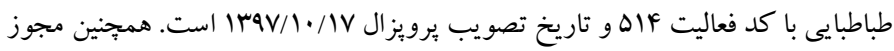

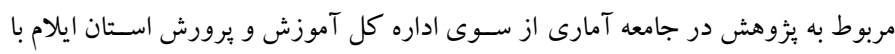

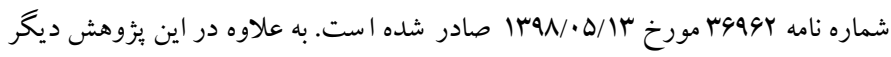

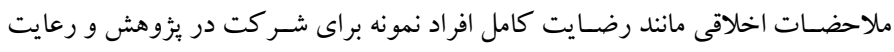
اصل رازدارى و محرمانه ماندن اطلاعات رعايت شده است. حامى مالى: اين مطالعه بدون حامى مالى و در قالب رساله دكتر انجام شده است. 


\section{References}

Amin Nasab V, Banijamali S A, Hatami H. (2019). The effectiveness of cognitive-motor learning training on social adjustment, motor skills and ADHD symptoms reduce in preschool children aged 5 and 6 years. Journal of psychological science, 17(72). 892-883. (Persian). [Link]

ghasemi K, kajbaf M B, qamarani A, torkan H. (2020). Effectiveness of domestic package of training executive functions specific for teachers on the signs of attention deficit and hyperactivity disorder (ADHD) of the children. Journal of psychological science, 90 (19). 763-755. (Persian). [Link]

Khorasani Zadeh A, Bahrami H, Ahadi H. (2018). Effectiveness of working memory training on recovery from behavioral symptoms (attentiondeficit and impulsivity) of children with attentiondeficit/hyperactivity. Journal of psychological science, 17(69). 544-537. (Persian). [Link]

Boustanzar, R., Rezayi, S. (2017). Developing intervention program focused and divided attention and investigating its effectiveness on working memory IQ in children with specific learning disorder. Journal of Learning Disabilities, 7(1), 725. (Persian). [Link]

rezaei E, Zaraii Zavaraki S, Hatami J, Ali Abadi K, Delavar A. (2017). Development of MOOCs Instructional Design Model Based on Connectivism Learning Theory. JEMD. 12 (1 and 2): 65-86. (Persian). [Link]

Au, J., Sheehan, E., Tsai, N., Duncan, G. J., Buschkuehl, M., \& Jaeggi, S. M. (2015). Improving fluid intelligence with training on working memory: a meta-analysis. Psychonomic bulletin \& review, 22(2), 366-377. [Link]

Basak, C., Boot, W. R., Voss, M. W., \& Kramer, A. F. (2008). Can training in a real-time strategy video game attenuate cognitive decline in older adults?. Psychology and aging, 23(4), 765-777. [Link]

Borella, E., Carretti, B., Riboldi, F., \& De Beni, R. (2010). Working memory training in older adults: evidence of transfer and maintenance effects. Psychology and aging, 25(4), 767-778. [Link]

Botvinick, M., \& Braver, T. (2015). Motivation and cognitive control: from behavior to neural mechanism. Annual review of psychology, 66(1), 83-113. [Link]

Bowden, S. C. (2013). Theoretical Convergence in Assessment of Cognition. Journal of
Psychoeducational Assessment, 31(2), 148-156. [Link]

Buschkuehl, M., \& Jaeggi, S. M. (2010). Improving intelligence: a literature review. Swiss medical weekly, 140(19-20), 266-272. [Link]

Carretti, B., Caldarola, N., Tencati, C., \& Cornoldi, C. (2014). Improving reading comprehension in reading and listening settings: the effect of two training programmes focusing on metacognition and working memory. The British journal of educational psychology, 84(2), 194-210. [Link]

Coloma, C. J., De Barbieri, Z., Quezada, C., Bravo, C., Chaf, G., \& Araya, C. (2020). The impact of vocabulary, grammar and decoding on reading comprehension among children with SLI: a longitudinal study. Journal of communication disorders, 86(1-2), 106002. [Link]

Dickens, W. T., \& Flynn, J. R. (2001). Heritability estimates versus large environmental effects: the IQ paradox resolved. Psychological review, 108(2), 346-369. [Link]

Flanagan, D \& Harrison, P. (2012). Contemporary intellectual assessment: Theories, tests, and issues. New York: Guilford Press.

Gottfredson, L., \& Saklofske, D.H. (2009). Intelligence: Foundations and Issues in Assessment. Canadian Psychology, 50(3), 183-195. [Link]

Halpern, D. F., Eliot, L., Bigler, R. S., Fabes, R. A., Hanish, L. D., Hyde, J., Liben, L. S., \& Martin, C. L. (2011). Education. The pseudoscience of singlesex schooling. Science (New York, N.Y.), 333(6050), 1706-1707. [Link]

Harrison, T. L., Shipstead, Z., Hicks, K. L., Hambrick, D. Z., Redick, T. S., \& Engle, R. W. (2014). Working memory training may increase working memory capacity but not fluid intelligence. Psychological science, 24(12), 2409-2419. [Link]

Heinzel, S., Schulte, S., Onken, J., Duong, Q. L., Riemer, T. G., Heinz, A., Kathmann, N., \& Rapp, M. A. (2014). Working memory training improvements and gains in non-trained cognitive tasks in young and older adults. Neuropsychology, development, and cognition. Section B, Aging, neuropsychology and cognition, 21(2), 146-173. [Link]

Jacky, au;Sheehan, Ellen; Tsai, Nancy; Duncan, Greg J.; Buschkuehl, Martin \& Jaeggi, Susanne M (2015). Improving fluid intelligence with training on working memory: a meta-analysis. Psychon Bull Rev. 22(2):366-77. [Link]

Jaeggi, S. M., Buschkuehl, M., Jonides, J., \& Perrig, W. J. (2008). Improving fluid intelligence with training 
on working memory. Proceedings of the National Academy of Sciences of the United States of America, 105(19), 6829-6833. [Link]

Jensen, A. (1969). How Much Can We Boost IQ and Scholastic Achievement. Harvard Educational Review; 39(1), 1-123. [Link]

Johnson, W., \& Bouchard, T. (2011). The MISTRA Data: Forty-Two Mental Ability Tests in Three Batteries. Intelligence, 39(2), 82-88. [Link]

Kan K, Kievit R, Dolan C, der M (2011). On the interpretation of the $\mathrm{CHC}$ factor Gc. Intelligence; 39(5), 292-302. [Link]

Kane, M. J., Hambrick, D. Z., \& Conway, A. (2005). Working memory capacity and fluid intelligence are strongly related constructs: comment on Ackerman, Beier, and Boyle (2005). Psychological bulletin, 131(1), 66-71. [Link]

Karageorgos, P., Richter, T., Haffmans, M., Schindler, J., \& Naumann, J. (2020). The role of wordrecognition accuracy in the development of wordrecognition speed and reading comprehension in primary school: A longitudinal examination. Cognitive Development, 56(4), 100949. [Link]

Kaufman, A. (2009). IQ testing 101. New York: Springer. Keith, T., \& Reynolds, M. (2010). Cattell-Horn-Carroll Abilities and Cognitive Tests: What We've Learned from 20 Years of Research. Psychology in the Schools, 47(7), 635-650. [Link]

Lynn, R. (2008). The Global Bell Curve: Race, IQ, and Inequality. Augusta: Washington Summit Publishers.

Mc Grew. (2005). the Cattell-Horn-Carroll Theory of Cognitive Abilities: Past, Present, and Future. In D. P. Flanagan \& P. L. Harrison (Eds.), Contemporary Intellectual Assessment: Theories, Tests, and Issues. New York: Guilford Press.

Mcardle, J., Ferrer-Caja, E., Hamagami, F., \& Woodcock, R. (2002). Comparative longitudinal structural analyses of the growth and decline of multiple intellectual abilities over the life span. Developmental psychology, 38(1), 115-42. [Link]

Nickerson, R. (2020). Developing Intelligence through Instruction. Cambridge Handbooks in Psychology. 2 ed. Cambridge: Cambridge University Press.

Nisbett, R., Aronson, J., Blair, C., Dickens, W., Flynn, J., Halpern, D., \& Turkheimer, E. (2012). Intelligence: new findings and theoretical developments. The American psychologist, 67(2), 130-59. [Link]

Reynolds, M., \& Turek, J.J. (2012). A dynamic developmental link between verbal comprehensionknowledge $(\mathrm{Gc})$ and reading comprehension: verbal comprehension-knowledge drives positive change in reading comprehension. Journal of school psychology, 50(6), 841-63. [Link]

Ritchie, S., \& Tucker-Drob, E. (2018). How Much Does Education Improve Intelligence? A Meta-Analysis. Psychological Science, 29(8), 1358 - 1369. [Link]

Rubio, D., Berg-Weger, M., Tebb, S., Lee, E.S., \& Rauch, S. (2003). Objectifying Content Validity: Conducting a Content Validity Study in Social Work Research. Social Work Research, 27(2), 94104. [Link]

Rushton, J., \& Jensen, A. (2010). The rise and fall of the Flynn Effect as a reason to expect a narrowing of the Black-White IQ gap. Intelligence, 38(2), 213219. [Link]

Rushton, P. (2000). Race, evolution, and behavior: A life history perspective. Port Huron: Charles Darwin Research Institute.

Stankov, L. (1986). Kvashchev's experiment: Can we boost intelligence? Intelligence; 10(3), 209-30. [Link]

Stemler, S.E., Grigorenko, E., Jarvin, L., \& Sternberg, R. (2006). Using the theory of successful intelligence as a basis for augmenting AP exams in Psychology and Statistics. Contemporary Educational Psychology, 31(3), 344-376. [Link]

Sternberg, R. (2002). Raising the Achievement of All Students: Teaching for Successful Intelligence. Educational Psychology Review, 14(4), 383-393. [Link]

Sternberg, R. (2015). Successful intelligence: A model for testing intelligence beyond IQ tests. European Journal of Education and Psychology 8(2), 76-84. [Link]

Sternberg, R. (2020). The Augmented Theory of Successful Intelligence. Cambridge Handbooks in Psychology. 2 ed. Cambridge: Cambridge University Press. [Link]

Sternberg, R., \& Grigorenko, E. (2003). Teaching for Successful Intelligence: Principles, Procedures, and Practices. Journal for the Education of the Gifted, 27(2-3), 207 - 228. [Link]

Westby, C. (2020). Growth Mindsets: Ideas from Carol Dweck. Word of Mouth, 31(5), 1 - 3. [Link]

Sternberg R J., Kaufman J C \& Grigorenko, E L. Applied Intelligence. New York: Cambridge University Press; 2008.

Wechsler D. WISC-V Technical and interpretive manual. Bloomington: Pearson; 2014. 